\title{
Does Treewidth Help in Modal Satisfiability? (Extended Abstract)
}

\author{
M. Praveen \\ The Institute of Mathematical Sciences, Chennai, India
}

\begin{abstract}
Many tractable algorithms for solving the Constraint Satisfaction Problem (CsP) have been developed using the notion of the treewidth of some graph derived from the input CsP instance. In particular, the incidence graph of the CsP instance is one such graph. We introduce the notion of an incidence graph for modal logic formulae in a certain normal form. We investigate the parameterized complexity of modal satisfiability with the modal depth of the formula and the treewidth of the incidence graph as parameters. For various combinations of Euclidean, reflexive, symmetric and transitive models, we show either that modal satisfiability is FPT, or that it is W[1]-hard. In particular, modal satisfiability in general models is FPT, while it is W[1]-hard in transitive models. As might be expected, modal satisfiability in transitive and Euclidean models is FPT.
\end{abstract}

\section{Introduction}

Treewidth as a parameter has been very successful in obtaining Fixed Parameter Tractable (FPT) algorithms for many classically intractable problems. One such class of problems is constraint satisfaction and closely related problems like satisfiability in propositional logic and the homomorphism problem [8, 30. There have been recent extensions to quantified constraint satisfaction [6, 27. In such problems, treewidth is used as a measure of modularity inherent in the given problem instance and algorithms make use of the modularity to increase their efficiency. Understanding the extent to which treewidth can be stretched in such problems is an active area of research 24, 15. This work explores the applicability of such techniques to modal satisfiability.

Apart from having many applications (reasoning about knowledge [10], programming [28] and hardware verification [29] etc.), modal logics have nice computational properties 33, 14. Many tools have been built for checking satisfiability of modal formulae 21, 26, despite being intractable in the classical sense (PSPACEcomplete or NP-complete in most cases). Complexity of modal logic decision problems is well studied [23, 17. 16. Another motivation for this work is to strengthen the complexity classification of modal logics through the refined analysis offered by parameterized complexity.

Our results: It is known that any modal logic formula can be effectively converted into a Conjunctive Normal Form (CNF) [9, 20. Given a modal logic formula in CNF, we associate a graph with it. Restricted to propositional CNF formulae (which are modal formulae with modal depth 0 ), this graph is precisely the incidence graph associated with propositional CNF formulae (see [30] for details). We prove that

1. with the treewidth of the graph and the modal depth of the formula as parameters, satisfiability in general models is FPT,

2. with treewidth and modal depth as parameters, satisfiability in transitive models is W[1]-hard and

3. with treewidth as the parameter, satisfiability in models that are Euclidean 1 and any combination of reflexive, symmetric and transitive is FPT.

Since modal formulae of modal depth 0 contain all propositional formulae, bounding modal depth alone will not give FPT results (unless PtimE=NP). The main idea behind our FPT results is to express satisfiability of a modal formula in Monadic Second Order (MSO) logic over the formula's associated graph and then apply Courcelle's theorem 7. Modal formulae with low treewidth are quite powerful, capable of encoding complex problems (see the conclusion for relevant pointers). On the other hand, modal formulae with low treewidth contain propositional CNF formulae of low treewidth, which arise naturally in many practical applications. See [12, Section 1.4] and references therein for some context on this.

Related work: In [16, Halpern considers the effect of bounding different parameters (such as the number of propositional variables, modal depth etc., but not treewidth) on complexity. In [25, Nguyen shows that satisfiability of many modal logics reduce to PTIME under the restriction of Horn fragment and bounded modal depth. In [1], Achilleos et. al. consider parameterized complexity of modal satisfiability in general models with the number of propositional variables and other structural aspects (but not treewidth) as

\footnotetext{
${ }^{1}$ A binary relation $\mapsto$ is Euclidean if $\forall x, y, z, x \mapsto y$ and $x \mapsto z$ implies $y \mapsto z$.
} 
parameters. In [2, Adler et. al. associate treewidth with First Order (FO) formulae and use it to obtain a FPT algorithm for model checking.

The Complexity of satisfiability of modal logics follow a pattern. In [18, Halpern et. al. prove that with the addition of Euclidean property, complexity of (infinitely) many modal logics drop from PsPACE-hard to NP-complete. [19] is another work in this direction. Similar pattern is observed in graded modal logics 22. With treewidth and modal depth as parameters, our results indicate similar behaviour in the world of parameterized complexity - satisfiability in transitive models is W[1]-hard, while satisfiability in Euclidean and transitive models is FPT, even with treewidth as the only parameter. However, more work is needed in this direction. First, the results in [18, 19] hold for infinitely many cases while we consider only a few fixed cases. Second, satisfiability in general models is PSPACE-complete and drops to NP-complete with the addition of Euclidean property. In our setting, satisfiability in general models is already FPT (but see conclusion for a discussion about why satisfiability in general models is not FPT unless PTIME=NP, when treewidth is the only parameter).

\section{Preliminaries}

Let $\mathbb{N}$ denote the set of natural numbers. For $k \in \mathbb{N}$, we denote the set $\{1, \ldots, k\}$ by $[k]$. We use standard notation about parameterized complexity like FPT algorithms, FPT reductions and W[1]-hardness from [13. We will also use notation and definitions of relational structures and their tree decompositions from [13: a relational vocabulary $\tau$ is a set of relation symbols. Each relation symbol $R$ has an arity $\operatorname{arity}(R) \geq 1$. A $\tau$-structure $\mathcal{S}$ consists of a set $D$ called the domain and an interpretation $R^{\mathcal{S}} \subseteq D^{\text {arity }(R)}$ of each relation symbol $R \in \tau$. A graph is an $\{E\}$-structure, where $E$ is a binary edge relation. A tree is a graph without cycles. A path decomposition is a tree decomposition [13, Definition 11.23] whose underlying tree is a path. The pathwidth of a structure is the minimum of the widths of all path decompositions. It is known that computing optimal tree and path decompositions of a relational structure is FPT when parameterized by treewidth; cf. [13, Corollary 11.28] and [5].

Courcelle's theorem ([13, Theorem 11.37]) states that given a relational structure and a MSO sentence, checking whether the MSO sentence is true in the structure is FPT when parameterized by the treewidth of the structure and the length of the sentence.

We use standard notation for modal logic from [3]: well formed modal logic formulae are defined by the grammar $\phi::=q \in \Phi|\perp| \neg \phi|\phi \vee \psi| \diamond \phi \mid \square \phi$, where $\Phi$ is a set of propositional variables. A Kripke model for the basic modal language is a triple $\mathcal{M}=(W, \mapsto, V l)$, where $W$ is a set of worlds, $\mapsto$ is a binary accessibility relation on $W$ and $V l: W \times \Phi \rightarrow\{\top, \perp\}$ is a valuation function. For $w, v \in W$, if $w \mapsto v$, $v$ is said to be a successor of $w$. The pair $(W, \mapsto)$ is called the frame $\mathcal{A}$ underlying $\mathcal{M}$. If $\mapsto$ is reflexive, then $\mathcal{A}$ and $\mathcal{M}$ are said to be a reflexive frame and a reflexive model respectively. Similar nomenclature is followed for other properties of $\mapsto$. The relation $\mapsto$ is Euclidean if for all $w_{1}, w_{2}, w_{3}, w_{1} \mapsto w_{2}$ and $w_{1} \mapsto w_{3}$ implies $w_{2} \mapsto w_{3}$. We denote the fact that a modal formula $\phi$ is satisfied at a world $w$ in a model $\mathcal{M}$ by $\mathcal{M}, w \models \phi$. For $q \in \Phi, \mathcal{M}, w \models q$ iff $V l(w, q)=\top$. Negation $\neg$ and disjunction $\vee$ are treated in the standard way. For any formula $\phi, \mathcal{M}, w \models \diamond \phi(\mathcal{M}, w \models \square \phi)$ iff some (all) successor(s) $v$ of $w$ satisfy $\mathcal{M}, v \models \phi$. A modal formula $\phi$ is satisfiable if there is a model $\mathcal{M}$ and a world $w$ in $\mathcal{M}$ such that $\mathcal{M}, w \models \phi$. A world $w^{\prime}$ is said to be reachable from $w$ if there are worlds $w_{1}, w_{2}, \ldots, w_{m}$ such that $w \mapsto w_{1} \mapsto \cdots \mapsto w_{m} \mapsto w^{\prime}$. It is well known that if some modal formula is satisfied at some world $w$ in some Kripke model, discarding worlds not reachable from $w$ does not affect satisfiability [3, Proposition 2.6]. Henceforth, if some modal formula is satisfied at some world $w$ in some Kripke model $\mathcal{M}$, we will assume that $\mathcal{M}$ consists of only those worlds reachable from $w$. Satisfiability in general, reflexive and transitive models are all PSPACE-complete 23], while in equivalence models, it is NP-complete [23.

The modal depth $\operatorname{md}(\phi)$ of a modal formula $\phi$ is inductively defined as follows. $\operatorname{md}(q)=\operatorname{md}(\perp)=0$. $\operatorname{md}(\neg \phi)=\operatorname{md}(\phi) \cdot \operatorname{md}(\phi \vee \psi)=\max \{\operatorname{md}(\phi), \operatorname{md}(\psi)\} \cdot \operatorname{md}(\diamond \phi)=\operatorname{md}(\square \phi)=\operatorname{md}(\phi)+1$. We will use the Conjunctive Normal Form (CNF) for modal logic defined in [20]:

$$
\begin{aligned}
\text { literal } & :=q|\neg q| \square \text { clause } \mid \diamond C N F \\
\text { clause } & :=\text { literal } \mid \text { clause } \vee \text { clause } \mid \perp \\
C N F & :=\text { clause } \mid C N F \wedge C N F
\end{aligned}
$$

where $q$ ranges over $\Phi$. Any arbitrary modal formula $\phi$ can be effectively transformed into CNF preserving satisfiability [9]. A $C N F$ is a conjunction of clauses and a clause is a disjunction of literals. A literal is either a propositional variable, a negated propositional variable or a formula of the form $\square$ clause or $\diamond C N F$. If one 
of the many literals in a clause is $\perp$, then $\perp$ can be ignored without affecting satisfiability. A literal of the form $\diamond \perp$ can similarly be ignored. However, a clause that has $\perp$ as the only literal cannot be ignored since $\square \perp$ is satisfied by a world in some Kripke model iff that world has no successors. Henceforth, we will assume that $\perp$ occurs only inside sub-formulae of the form $\square \perp$.

Suppose $\phi$ is a modal formula in CNF. If $\phi$ is of the form clause $_{1} \wedge$ clause $_{2} \wedge \cdots \wedge$ clause $_{\mathrm{m}}$, then clause $_{1}$, clause $_{2}, \ldots$, clause $_{\mathrm{m}}$ and all literals appearing in these clauses are said to be at level $\mathrm{md}(\phi)$. If $\square$ clause $_{1}$ is a literal at some level $i$, then clause $_{1}$ and all literals occurring in clause $_{1}$ are said to be at level $i-1$. If $\triangle C N F$ is a literal at some level $i$ and $C N F$ is of the form clause $_{1} \wedge$ clause $_{2} \wedge \cdots \wedge$ clause $_{\mathrm{m}^{\prime}}$, then clause $_{1}$, clause $_{2}, \cdots$, clause $_{\mathrm{m}^{\prime}}$ and all literals appearing in these clauses are said to be at level $i-1$. Note that a single propositional variable can occur in the form of a literal at different levels. The concept of level is similar to the concept of distance defined in [26. The process of checking satisfiability we describe in section 3 can be considered a variant of the level-based bottom-up algorithm given in [26], which is also implicitly used in [1, Theorem 5]. It requires more work and combination of other ideas to prove that this process can be formalized in MSO logic.

\section{Modal satisfiability in general models}

In this section, we will associate a relational structure with a modal CNF formula. We show that checking satisfiability of a modal CNF formula is FPT, parameterized by modal depth and the treewidth of the associated relational structure. We begin with an example modal CNF formula.

Consider the modal CNF formula $\{\neg q \vee \square[r \vee \neg s]\} \wedge\{q \vee \diamond \perp\} \wedge\{r \vee \diamond[\neg s]\} \wedge\{\neg r \vee \diamond[(t \vee \neg s) \wedge(r)]\}$. Its modal depth is 1 and has 4 clauses at level 1. Figure 1 shows a graphical representation of this formula, which is very similar to the formula's syntax tree. The 4 clauses at level 1 are represented by $e_{1}, e_{2}, e_{3}$ and $e_{4}$. $e_{1}$ represents the clause $\{\neg q \vee \square[r \vee \neg s]\}$. Since $\neg q$ occurs as a literal in this clause, there is a dotted arrow from $e_{1}$ to $q . \square[r \vee \neg s]$ (represented by $e_{9}$ ) also occurs as a literal in clause $e_{1}$ and hence there is an arrow from $e_{1}$ to $e_{9} . e_{4}$ represents the fourth clause at level 1 , which contains $\diamond[(t \vee \neg s) \wedge(r)]$ as a literal. This $\checkmark C N F$ formula is represented by $e_{10}$. The two clauses $(t \vee \neg s)$ and $(r)$ are represented by $e_{7}$ and $e_{8}$ respectively and are connected to $e_{10}$ by arrows. The propositional variable $r$ occurs as literal at 2 levels, indicated as $L v_{0}$ and $L v_{1}$.

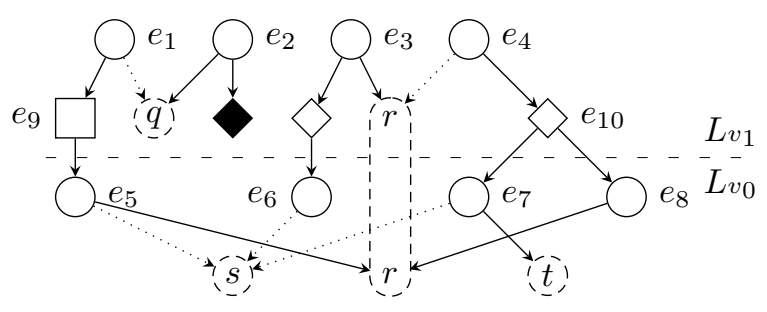

Fig. 1. Relational structure associated with the modal formula $\{\neg q \vee \square[r \vee \neg s]\} \wedge\{q \vee \diamond \perp\} \wedge\{r \vee \diamond[\neg s]\} \wedge$ $\{\neg r \vee \diamond[(t \vee \neg s) \wedge(r)]\}$

Now we will formalize the above example. The intuition behind the following definition is to represent all clauses and literals of a modal CNF formula by the domain elements of a relational structure. Binary relations are used to indicate which literals occur in which clause (and which clauses occur in which literal). Unary relations are used to indicate which elements represent literals and which elements represent clauses. This will enable us to reason about clauses, literals and their dependencies using MSO formulae over the relational structure.

Definition 3.1. Given a modal CNF formula $\phi$, we associate with it a relational structure $\mathcal{S}(\phi)$. It will have one domain element for every clause in $\phi$. It will have one domain element for every literal of the form $\square$ clause or $\checkmark C N F$ in $\phi$. It will also have one domain element for every propositional variable used in $\phi$. There are no domain elements representing the propositional constant $\perp$. They will be handled as special cases.

The relational structure will have two binary relations $O_{c}$ (occurs) and $\overline{O_{c}}$ (occurs negatively). $\overline{O_{c}}\left(e_{1}, e_{2}\right)$ iff $e_{1}$ represents a clause and $e_{2}$ represents a propositional variable occurring negated as a literal in the 
clause represented by $e_{1}$. If $e_{1}$ represents a clause, then $O_{c}\left(e_{1}, e_{2}\right)$ iff $e_{2}$ represents a literal (occurring in the clause represented by $e_{1}$ ) of the form $\square$ clause, $\triangle C N F$ or a non-negated propositional variable. If $e_{1}$ represents a literal of the form $\square$ clause, then $O_{c}\left(e_{1}, e_{2}\right)$ iff $e_{2}$ represents the corresponding clause. If $e_{1}$ represents a literal of the form $\diamond C N F$, then $O_{c}\left(e_{1}, e_{2}\right)$ iff $e_{2}$ represents a clause in the corresponding CNF. Finally, the following unary relations are present:

$\mathrm{Cl}$ : contains all domain elements representing clauses

Lt : all domain elements representing literals

$U$ : all literals of the form $\square \perp$

$B \square$ : all literals of the form $\square$ clause

$D \diamond:$ all literals of the form $\diamond C N F$

$\left(L v_{i}\right)_{0 \leq i \leq m d(\phi)}$ : all clauses and literals at level $i$

For clauses and literals of the form $\square$ clause or $\diamond C N F$, there is one domain element for every occurrence of the clause or literal. For example, if the literal $\diamond\left(q_{1} \wedge q_{2}\right)$ occurs in two different positions of a big formula $\phi$, the two occurrences will be represented by two different domain elements in $\mathcal{S}(\phi)$. In contrast, different occurrences of a literal that is just a propositional variable will be represented by the same domain element. In the rest of the paper, whenever we refer to the treewidth of a modal CNF formula $\phi$, we mean the treewidth of $\mathcal{S}(\phi)$.

If $e_{1}$ represents a clause, $O_{c}\left(e_{1}, e_{2}\right)$ means that the clause represented by $e_{1}$ can be satisfied by satisfying the literal represented by $e_{2} . \overline{O_{c}}\left(e_{1}, e_{2}\right)$ means that the clause represented by $e_{1}$ can be satisfied by setting the propositional variable represented by $e_{2}$ to false.

If $C_{\ell_{0}} \subseteq C l \cap L v_{0}$ is a subset of domain elements representing clauses at level 0 , let $C N F\left(\mathrm{C}_{\ell_{0}}\right)$ be the modal CNF formula that is the conjunction of clauses represented by domain elements in $C_{\ell_{0}}$. We will now see how to check satisfiability of $C N F\left(\left\{\mathrm{e}_{7}, \mathrm{e}_{8}\right\}\right)$ in Fig. 1 and describe the generalization of this process given in (11) below. We use $c_{\ell}$ and $l_{t}$ for first order variables intended to represent clauses and literals respectively. First of all, there must be a subset $T_{r_{0}} \subseteq\{r, s, t\}=L t \cap L v_{0}$ that will be set to $T$, as written in the beginning of (11). Then, we must check that this assignment satisfies each clause $c_{\ell}$ in $C_{\ell_{0}}$, written as $\forall c_{\ell} \in C_{\ell_{0}}$ in (1D). To check that the clause represented by $e_{7}$ is satisfied, either a positively occurring literal like $t$ must be set to $T$ and hence in $T_{r_{0}}$ (written as " $\exists l t \in T_{r_{0}}: O_{c}\left(c_{\ell}, l_{t}\right)$ " in (10) or a negatively occurring literal like $s$ must be set to $\perp$ and hence not in $\operatorname{Tr}_{0}$ (" $\exists l t \in\left(L t \cap L v_{0}\right) \backslash T_{r_{0}}: \overline{O_{c}}\left(c_{\ell}, l_{t}\right)$ " in (1D)). A similar argument applies to $e_{8}$ as well.

$$
\begin{aligned}
& \xi[0]\left(C_{\ell_{0}}\right) \triangleq \exists T_{r_{0}} \subseteq\left(L t \cap L v_{0}\right): \forall c_{\ell} \in C_{\ell_{0}}: \\
& {\left[\left(\exists l t \in T_{r_{0}}: O_{c}\left(c_{\ell}, l_{t}\right)\right) \vee\left(\exists l t \in\left(L t \cap L v_{0}\right) \backslash T_{r_{0}}: \overline{O_{c}}\left(c_{\ell}, l_{t}\right)\right)\right]} \\
& \xi[i]\left(C_{\ell_{i}}\right) \triangleq \exists T r_{i} \subseteq\left(L t \cap L v_{i}\right): \forall c_{\ell} \in C_{\ell_{i}}: \\
& {\left[\left(\exists l_{t} \in T_{r_{i}}: O_{c}\left(c_{\ell}, l_{t}\right)\right) \vee\left(\exists l t \in\left(L t \cap L v_{i}\right) \backslash T_{r_{i}}: \overline{O_{c}}\left(c_{\ell}, l_{t}\right)\right)\right]} \\
& \wedge\left[C_{m_{i-1}}=\left\{c e^{\prime} \in\left(C l \cap L v_{i-1}\right) \mid \exists l t^{\prime} \in T r_{i} \cap B \square, O c\left(l t^{\prime}, c e^{\prime}\right)\right\} \Rightarrow\right. \\
& \forall l_{t} \in T_{r_{i}} \cap D \diamond: D m_{i-1}=\left\{c_{l} \in\left(C_{l} \cap L v_{i-1}\right) \mid O_{c}\left(l_{t}, c_{l}\right)\right\} \Rightarrow \\
& \left.\xi[i-1]\left(D m_{i-1} \cup C m_{i-1}\right)\right]
\end{aligned}
$$

Checking satisfiability at higher levels is slightly more complicated. Suppose $C_{\ell_{i}} \subseteq C l \cap L v_{i}$ is a subset of clauses at level $i$. We will take $C_{\ell_{1}}=\left\{e_{1}, e_{3}, e_{4}\right\}$ from Fig. 1 as an example. If some world $w$ in some Kripke model $\mathcal{M}$ satisfies $C N F\left(\mathrm{C}_{\ell_{1}}\right)$, there must be some subset $T_{r_{1}}$ of literals at level 1 satisfied at $w$ (" $\exists T r_{i} \subseteq\left(L t \cap L v_{i}\right)$ " in (2)). As before, we check that for every clause represented in $C_{\ell_{1}}$ (" $\forall c_{\ell} \in C_{\ell_{i}}$ " in (2)), there is either a positively occurring literal in $T_{r_{1}}$ (" $\exists l t \in T_{r_{i}}: O c\left(C_{\ell}, l_{t}\right)$ " in (2)) or a negatively occurring literal not in $T_{r_{1}}$ (" $\exists l t \in\left(L t \cap L v_{i}\right) \backslash T_{r_{i}}: \overline{O_{c}}\left(c_{\ell}, l_{t}\right)$ " in (2) ). Next, we must check that the literals we have chosen to be satisfied at $w$ (by putting them into $T_{r}$ ) can actually be satisfied. Suppose $T_{r_{1}}$ was $\left\{e_{9}, q, r, e_{10}\right\}$. Since $e_{9}$ represents a literal of the form $\square$ clause (with the clause represented by domain element $e_{5}$ ), we are committed to satisfy the clause represented by $e_{5}$ in any world succeeding $w$. Let $C_{m_{0}}=\left\{e_{5}\right\}$ be the set of clauses occurring at level 0 that we have committed to as a result of choosing corresponding $\square$ clause literals to be in $T_{r_{1}}$ ("C $C_{m_{i-1}}=\left\{c \ell^{\prime} \in\left(C l \cap L v_{i-1}\right) \mid \exists l t^{\prime} \in T r_{i} \cap B \square, O c\left(l t^{\prime}, c \ell^{\prime}\right)\right\}$ " in (2)). Now, since we have also chosen $e_{10}$ to be in $T r_{1}$ and $e_{10}$ represents a $\diamond C N F$ formula, there is a demand to create a world $w^{\prime}$ that succeeds $w$ and satisfies the corresponding CNF formula. We have to check that every such demand in $T r_{1}$ can be satisfied (" $\forall l t \in T r_{i} \cap D \diamond$ " in (2)) by creating successor worlds. In case of the demand created by $e_{10},\left\{e_{7}, e_{8}\right\}=D_{m_{0}}$ is the set of clauses in the demanded $C N F$ formula ( "D $m_{i-1}=\left\{c_{\ell} \in\left(C_{l} \cap L v_{i-1}\right) \mid O_{c}\left(l t, c_{\ell}\right)\right\} "$ in (2)). Our aim now is to create a successor world $w^{\prime}$ in which all clauses represented in $D_{m_{0}}$ are satisfied. However, $w^{\prime}$ is a successor world and we have already committed 
to satisfying all clauses represented in $C_{m_{0}}$ in all successor worlds. Hence, we actually check if the clauses represented in $C_{m_{0}} \cup D m_{0}$ are satisfiable by inductively invoking $\xi[0]\left(D m_{0} \cup C_{m_{0}}\right)$ (" $\xi[i-1]\left(D m_{i-1} \cup C m_{i-1}\right)$ " in (2) ).

For the sake of clarity, we have skipped handling literals of the form $\square \perp$ in the above discussion. They will be handled in the formal arguments that follow.

Lemma 3.2. The property $\xi[i]\left(C_{\ell_{i}}\right)$ can be written in a MSO logic formula of size linear in $i$. If $\phi$ is any modal formula in $C N F$ and $C_{\ell_{i}}$ is any subset of domain elements representing clauses at level $i$, then $\operatorname{CNF}\left(\mathrm{C}_{\ell_{\mathrm{i}}}\right)$ is satisfiable iff $\xi[i]\left(C_{\ell_{i}}\right)$ is true in $\mathcal{S}(\phi)$.

Proof. We will prove the first claim by induction on $i$. Formula (3) below is same as (1) written in formal MSO syntax. (4) is a formal MSO statement of (2) and two additional conditions for handling literals of the form $\square \perp$ and $\diamond \perp$. We will prove that the length $|\xi[i]|$ of $\xi[i]$ is linear in $i$. Let $c$ be the length of $\xi[i]$ without length of $\xi[i-1]$ counted. As can be seen, $|\xi[0]| \leq c$. Inductively assume that $|\xi[i-1]| \leq i c$. Then, $|\xi[i]|=c+|\xi[i-1]|$. Hence, $|\xi[i]| \leq c+i c=c(i+1)$.

We will now prove the second claim by induction on $i$.

Base case $i=0$ : The modal formula $C N F\left(\mathrm{C}_{\ell_{0}}\right)$ is a propositional CNF formula. Suppose $\xi[0]\left(C_{\ell_{0}}\right)$ is true in $\mathcal{S}(\phi)$. Hence, there is a subset $T_{r_{0}}$ of domain elements that satisfy the last four conditions of $\xi[0]$ defined in (3). The second condition $\forall x\left(T r_{0}(x) \Rightarrow\left(L t(x) \wedge L v_{0}(x)\right)\right)$ ensures that all domain elements in $T r_{0}$ are also in $L t$ and $L v_{0}$. Hence, all domain elements in $T r_{0}$ represent literals at level 0 . Since the only literals at level 0 are propositional variables or their negations, $T_{r_{0}}$ is in fact a subset of propositional variables. Consider the Kripke model $\mathcal{M}$ with a single world $w$ at which, all propositional variables in $T r_{0}$ are set to $T$ and all others are set to $\perp$. We will now prove that all clauses represented in $C_{\ell_{0}}$ are satisfied in $w$. Let $c_{\ell}$ be some element in $C_{\ell_{0}}$ representing some clause. Since $C_{\ell_{0}}\left(c_{\ell}\right)$ is true and $\mathcal{S}(\phi)$ satisfies the last three conditions of $\xi[0]$, we have that either $\exists l t\left(T_{r_{0}}\left(l_{t}\right) \wedge O_{c}\left(c_{\ell}, l_{t}\right)\right)$ or $\exists l_{t}\left(L t(l t) \wedge L v_{0}\left(l_{t}\right) \wedge \neg T_{r_{0}}\left(l_{t}\right) \wedge \overline{O_{c}}\left(c_{\ell}, l_{t}\right)\right)$ is true in $\mathcal{S}(\phi)$. In the first case, $O_{c}\left(c_{\ell}, l_{t}\right)$ means that $l_{t}$ is a positively occurring literal in the clause $c_{\ell}$ and $T_{r_{0}}\left(l_{t}\right)$ means that $l_{t}$ is in $T r_{0}$ (and hence it is set to $T$ in $w$, satisfying clause $c_{\ell}$ ). In the second case, $\overline{O_{c}}\left(c_{\ell}, l_{t}\right) \wedge L t\left(l_{t}\right) \wedge L_{v_{0}}\left(l_{t}\right)$ means that $l_{t}$ is a literal negatively occurring in clause $c_{\ell}$ and $\neg T r_{0}\left(l_{t}\right)$ means that $l_{t}$ is not in $T r_{0}$ (and hence it is set to $\perp$ in $w$, again satisfying clause $c \ell$ ).

Now suppose that there is a Kripke model $\mathcal{M}$ and a world $w$ such that $\mathcal{M}, w \models C N F\left(\mathrm{C}_{\ell_{0}}\right)$. We will prove that $\xi[0]\left(C_{\ell_{0}}\right)$ is true in $\mathcal{S}(\phi)$. The first requirement is to find a suitable subset $T_{r_{0}}$ of domain elements. We will set $T_{r_{0}}$ to be the set of precisely those domain elements that represent propositional variables occurring at level 0 and set to $T$ in the world $w$. This will ensure that the condition $\forall x\left(T r_{0}(x) \Rightarrow\left(L t(x) \wedge L v_{0}(x)\right)\right)$ in $\xi[0]$ is satisfied. Now we have to prove that last three conditions of $\xi[0]$ are satisfied. So let $c_{\ell} \in C_{\ell_{0}}$ be any domain element so that it satisfies $C_{\ell_{0}}\left(c_{\ell}\right)$. We have to now prove that this $c_{\ell}$ satisfies one of the last two conditions of $\xi[0]$. Since $c_{\ell} \in C_{\ell_{0}}$, it represents a clause in $\phi$ occurring at level 0 . Since $\mathcal{M}, w \models C N F\left(\mathrm{C}_{\ell_{0}}\right)$, the clause represented by $c_{\ell}$ is satisfied in $w$. Hence there is either a positively occurring propositional variable set to $T$ in $w$ (so that it is in $T_{r_{0}}$, thus satisfying $\left.\exists l_{t}\left(\operatorname{Tr}_{0}\left(l_{t}\right) \wedge O_{c}\left(c_{\ell}, l_{t}\right)\right)\right)$ or a negatively occurring propositional variable set to $\perp$ in $w$ (so that it is not in $T_{r_{0}}$, thus satisfying $\exists l t\left(L t(l t) \wedge L v_{0}(l t) \wedge \neg \operatorname{Tr}_{0}(l t) \wedge \overline{O_{c}}\left(c_{\ell}, l_{t}\right)\right)$ ). This completes the base case.

$$
\begin{aligned}
& \xi[0]\left(C_{\ell_{0}}\right) \triangleq \exists T r_{0} \\
& \{ \\
& \forall x\left(\operatorname{Tr}_{0}(x) \Rightarrow\left(L t(x) \wedge L v_{0}(x)\right)\right) \\
& \wedge \forall c_{\ell} \quad C_{\ell_{0}}\left(c_{\ell}\right) \Rightarrow \\
& {[} \\
& \exists l t\left(T_{r_{0}}\left(l_{t}\right) \wedge O_{c}\left(c_{e}, l_{t}\right)\right) \\
& \vee \exists l t\left(L t\left(l_{t}\right) \wedge L v_{0}\left(l_{t}\right) \wedge \neg T_{r_{0}}\left(l_{t}\right) \wedge \overline{O_{c}}\left(c_{\ell}, l_{t}\right)\right) \\
& \text { \} } \\
& \text { ] }
\end{aligned}
$$

Induction step: Suppose $C_{\ell_{i}}$ is a subset of domain elements representing clauses occurring at level $i$ and $\xi[i]\left(C_{\ell_{i}}\right)$ is true in $\mathcal{S}(\phi)$. We will build a Kripke model $\mathcal{M}$ and prove that it has a world $w$ such that $\mathcal{M}, w \models$ $C N F\left(\mathrm{C}_{\ell_{\mathrm{i}}}\right)$. We will start with a single world $w$. Since $\xi[i]\left(C_{\ell_{i}}\right)$ is true in $\mathcal{S}(\phi)$, there must be a subset $T r_{i}$ of domain elements satisfying the last eleven conditions of $\xi[i]\left(C_{\ell_{i}}\right)$. The condition $\forall x\left(\operatorname{Tr}_{i}(x) \Rightarrow\left(L t(x) \wedge L v_{i}(x)\right)\right)$ ensures that all domain elements in this $T r_{i}$ represent literals occurring at level $i$. Let $P V\left(T r_{i}\right) \subseteq T r_{i}$ be those domain elements in $T r_{i}$ that represent propositional variables. Similarly, let $B L\left(T r_{i}\right)$ and $D L\left(T r_{i}\right)$ be the domain elements in $T_{r_{i}}$ representing literals of the form $\square$ clause and $\diamond C N F$ respectively. In our world $w$, 
set all propositional variables in $P V\left(T r_{i}\right)$ to $\top$ and set all others to $\perp$. Now, $w$ satisfies all literals represented in $P V\left(T r_{i}\right)$. We will later prove how to satisfy literals represented in $B L\left(T r_{i}\right)$ and $D L\left(T r_{i}\right)$ in the world $w$.

Now, assuming that all literals represented in $T_{r_{i}}$ are satisfied at $w$, we will prove that $\mathcal{M}, w \models C N F\left(\mathrm{C}_{\ell_{\mathrm{i}}}\right)$. This part of the proof is similar to the base case. If $c_{\ell}$ is any clause in $C_{\ell_{i}}$, it satisfies $C_{\ell_{i}}\left(c_{\ell}\right)$ and hence either $\exists l_{t}\left(T_{r_{i}}\left(l_{t}\right) \wedge O_{c}\left(c_{\ell}, l_{t}\right)\right)$ or $\exists l_{t}\left(L t(l t) \wedge L v_{i}\left(l_{t}\right) \wedge \neg T_{r_{i}}\left(l_{t}\right) \wedge \overline{O_{c}}\left(c_{\ell}, l_{t}\right)\right)$ is true in $\mathcal{S}(\phi)$. In the first case, a positively occurring literal at level $i$ is in $T_{r}$, and since all literals in $T_{r_{i}}$ are satisfied at $w$, the clause represented by $c_{\ell}$ is also satisfied at $w$. In the second case, a negatively occurring literal at level $i$ is not in $T r_{i}$. Since only propositional variables can occur negatively in clauses, we can in fact conclude that a negatively occurring propositional variable is not in $T r_{i}$. Since all propositional variables not in $T r_{i}$ are set to $\perp$ in $w$, the clause represented by $c_{\ell}$ is satisfied in $w$.

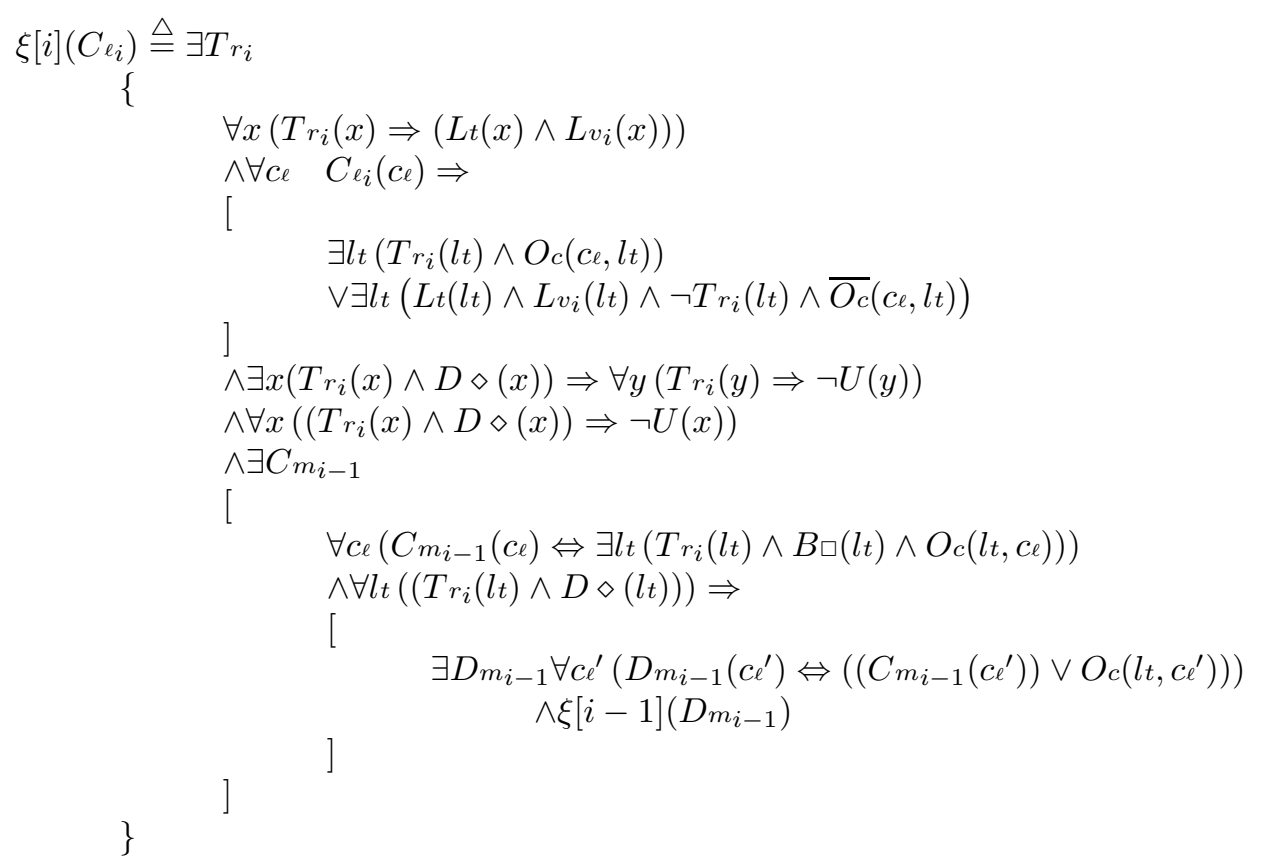

Now we will prove that literals represented in $B L\left(T r_{i}\right)$ and $D L\left(T r_{i}\right)$ can be satisfied in $w$ by adding appropriate successor worlds. First note that since $\forall x\left(\left(T_{r_{i}}(x) \wedge D \diamond(x)\right) \Rightarrow \neg U(x)\right)$ is true in $\mathcal{S}(\phi)$, no element $x$ in $T_{r_{i}}$ represents a literal of the form $\diamond \perp$ (since $U$ is the unary relation containing all domain elements representing literals of the form $\square \perp$ or $\diamond \perp)$. Second, note that since $\exists x\left(\operatorname{Tr}_{i}(x) \wedge D \diamond(x)\right) \Rightarrow$ $\forall y\left(T r_{i}(y) \Rightarrow \neg U(y)\right)$ is true in $\mathcal{S}(\phi)$, if $D L\left(T r_{i}\right)$ is not empty, then no element in $B L\left(T r_{i}\right)$ represents a literal of the form $\square \perp$. Therefore, we can hope to add a new successor for each literal represented by some element $l_{t}$ in $D L\left(T r_{i}\right)$, satisfying the $C N F$ formula in the literal represented in $l_{t}$ as well as all clauses in literals represented in $B L\left(T r_{i}\right)$. Now we will prove that this can actually be done.

Since $\xi[i]\left(C_{\ell_{i}}\right)$ is true in $\mathcal{S}(\phi)$, there is a subset $C_{m_{i-1}}$ of domain elements satisfying the last four conditions of $\xi[i]\left(C_{\ell_{i}}\right)$. The condition $\forall c_{\ell}\left(C_{m_{i-1}}\left(c_{\ell}\right) \Leftrightarrow \exists l_{t}\left(T_{r_{i}}\left(l_{t}\right) \wedge B \square\left(l_{t}\right) \wedge O_{c}\left(l_{t}, c_{\ell}\right)\right)\right)$ ensures that $C_{m_{i-1}}$ contains exactly those domain elements representing some clause clause $_{1}$ at level $i-1$ such that $\square$ clause $_{1}$ is a literal in $T_{r_{i}}$ (and hence $\square$ clause $_{1}$ is in $B L\left(T r_{i}\right)$ ). An element $l_{t}$ satisfies $T r_{i}\left(l_{t}\right) \wedge D \diamond\left(l_{t}\right)$ iff $l t \in D L\left(T r_{i}\right)$. Hence, the condition $\forall l_{t}\left(\left(\operatorname{Tr}_{i}\left(l_{t}\right) \wedge D \diamond\left(l_{t}\right)\right)\right) \Rightarrow[\cdots]$ ensures that the last two conditions of $\xi[i]\left(C_{\ell_{i}}\right)$ is true for every element $l_{t}$ in $T_{r_{i}}$ representing a literal of the form $\diamond C N F$ occurring at level $i$. Consider any one such element $l t$. The condition $\exists D m_{i-1} \forall c \ell^{\prime}\left(D m_{i-1}\left(c \ell^{\prime}\right) \Leftrightarrow\left(\left(C m_{i-1}\left(c \ell^{\prime}\right)\right) \vee O c\left(l t, c \ell^{\prime}\right)\right)\right)$ ensures that $D m_{i-1}$ contains exactly those elements representing some clause clause $_{1}$ (occurring at level $i-1$ ) such that $\square$ clause $_{1}$ is represented in $B L\left(T r_{i}\right)$ or clause $_{1}$ occurs in the $C N F$ formula in the $\diamond C N F$ literal represented by $l$. Since $\xi[i-1]\left(D_{m_{i-1}}\right)$ is true, we can apply the induction hypothesis and conclude that there is some Kripke model $\mathcal{M}^{\prime}$ and a world $w^{\prime}$ such that $\mathcal{M}^{\prime}, w^{\prime} \models C N F\left(\mathrm{Dm}_{\mathrm{i}-1}\right)$. Now, $w^{\prime}$ satisfies the $C N F$ formula in

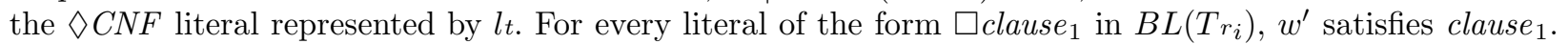
Now, we add the Kripke model $\mathcal{M}^{\prime}$ to $\mathcal{M}$ and make $w^{\prime}$ a successor of $w$. We repeat this procedure for every element $l_{t}$ in $D L\left(T r_{i}\right)$. Now, for every literal in $T r_{i}$ of the form $\diamond C N F$, there is a successor of $w$ that satisfies the corresponding $C N F$ formula (we have already proved that literals of the form $\diamond \perp$ will not be present in $\left.T r_{i}\right)$. For every literal in $T r_{i}$ of the form $\square$ clause, all successors of $w$ will satisfy the corresponding clause 
(we have already proved that if there is a literal of the form $\square \perp$ in $T r_{i}$, then $T r_{i}$ will not have any literals of the form $\triangle C N F$ and hence we will not add any successor worlds to $w$ ).

Now we will prove the other direction of the induction step. Suppose $C_{\ell_{i}}$ is a subset of domain elements representing clauses occurring at level $i$ and that there is a Kripke model $\mathcal{M}$ and a world $w$ such that $\mathcal{M}, w \models C N F\left(\mathrm{C}_{\ell_{\mathrm{i}}}\right)$. We will prove that $\xi[i]\left(C_{\ell_{i}}\right)$ is true in $\mathcal{S}(\phi)$. To begin with, we will choose $T_{r_{i}}$ to be the set of precisely those domain elements that represent literals occurring at level $i$ that are satisfied at $w$. If literals of the form $\square \perp$ occur at level $i$, then they will also be included in $T_{r_{i}}$ by definition if there are no successor worlds at $w$. Now we will prove that last eleven conditions of $\xi[i]\left(C_{\ell_{i}}\right)$ are true in $\mathcal{S}(\phi)$. The condition $\forall x\left(T_{r_{i}}(x) \Rightarrow\left(L t(x) \wedge L v_{i}(x)\right)\right)$ is true since all elements $x$ in $T_{r_{i}}$ are representing literals $(L t(x))$ at level $i\left(L v_{i}(x)\right)$. Next we will prove that the condition $\forall c_{\ell} C_{\ell_{i}}\left(c_{\ell}\right) \Rightarrow[\ldots]$ is true. Let $c_{\ell}$ be some arbitrary element in $C_{\ell_{i}}$. Since ce represents a clause that is satisfied at the world $w$, there must be either a positively occurring literal that is satisfied at $w$ (and hence the domain element representing that literal will be in $\operatorname{Tr}_{i}$, thus implying that $\exists l_{t}\left(\operatorname{Tr}_{i}\left(l_{t}\right) \wedge O_{c}\left(c_{\ell}, l_{t}\right)\right)$ is true in $\left.\mathcal{S}(\phi)\right)$ or there must be a negatively occurring literal that is not satisfied at $w$. In the latter case, since only propositional variables can occur negatively, we can in fact conclude that there is a negatively occurring propositional variable that is set to $\perp$ at $w$ (and hence not in $\left.T r_{i}\right)$, which implies that $\exists l t\left(L t\left(l_{t}\right) \wedge L v_{i}\left(l_{t}\right) \wedge \neg T r_{i}\left(l_{t}\right) \wedge \overline{O_{c}}\left(c_{\ell}, l t\right)\right)$ is true in $\mathcal{S}(\phi)$.

Next we will prove that the condition $\forall x\left(\left(T_{r_{i}}(x) \wedge D \diamond(x)\right) \Rightarrow \neg U(x)\right)$ is true. If any element $x$ is in $T_{r_{i}}$ $\left(\operatorname{Tr}_{i}(x)\right)$ and represents a literal of the form $\diamond C N F(D \diamond(x))$, then $x$ will not represent $\diamond \perp(\neg U(x))$ since $x$ represents a literal that is satisfied at $w$ and $\diamond \perp$ cannot be satisfied.

Next we will prove that the condition $\exists x\left(T r_{i}(x) \wedge D \diamond(x)\right) \Rightarrow \forall y\left(T_{r_{i}}(y) \Rightarrow \neg U(y)\right)$ is true. Suppose there is some element $x$ in $T r_{i}$ that represents a literal of the form $\diamond C N F\left(\exists x\left(T_{r_{i}}(x) \wedge D \diamond(x)\right)\right)$. Since the literal represented by $x$ is satisfied at $w$, there is a successor world in which the corresponding $C N F$ formula is satisfied. Since $w$ has successor worlds, it cannot satisfy $\square \perp$ and hence none of the elements in $T r_{i}$ represent literals of the form $\square \perp\left(\forall y\left(\operatorname{Tr}_{i}(y) \Rightarrow \neg U(y)\right)\right)$.

Finally, we will prove that the condition $\exists C m_{i-1}[\ldots]$ is true. Let us first construct the set $C m_{i-1}$. For any element $c_{\ell}\left(\forall c_{\ell}\right)$, we will put $c_{\ell}$ in $C_{m_{i-1}}$ iff $\left(C_{m_{i-1}}\left(c_{\ell}\right) \Leftrightarrow\right)$ there is some element $l_{t}(\exists l t)$ in $\operatorname{Tr}_{i}$ $\left(\operatorname{Tr}_{i}(l t)\right)$ representing a literal of the form $\square$ clause $\left(B \square\left(l_{t}\right)\right)$ such that $c_{\ell}$ represents the corresponding clause $\left(O c\left(l_{t}, c_{\ell}\right)\right)$. The condition $\forall c_{\ell}\left(C_{m_{i-1}}\left(c_{\ell}\right) \Leftrightarrow \exists l_{t}\left(T_{r_{i}}\left(l_{t}\right) \wedge B \square\left(l_{t}\right) \wedge O_{c}\left(l_{t}, c_{\ell}\right)\right)\right)$ is true in $\mathcal{S}(\phi)$ by construction. Next we will prove that the condition $\forall l_{t}\left(\left(\operatorname{Tr}_{i}\left(l_{t}\right) \wedge D \diamond\left(l_{t}\right)\right)\right) \Rightarrow[\ldots]$ is true. Suppose $l_{t}$ is any element in $T r_{i}$ representing a literal of the form $\diamond C N F_{1}(D \diamond(l t))$. Since $\diamond C N F_{1}$ is satisfied at the world $w$, there is a successor world $w^{\prime}$ that satisfies the corresponding $C N F_{1}$ formula. Let $D m_{i-1}$ be the set that includes some element $c \ell^{\prime}$ iff $\left(\forall c \ell^{\prime} D m_{i-1}\left(c \ell^{\prime}\right) \Leftrightarrow\right) c \ell^{\prime}$ is in the set $C m_{i-1}$ constructed above $\left(C m_{i-1}\left(c \ell^{\prime}\right)\right)$ or it represents a clause occurring in the $C N F_{1}$ formula contained in the $\diamond C N F_{1}$ literal represented by $l_{t}\left(O_{c}\left(l_{t}, c \ell^{\prime}\right)\right)$. $D m_{i-1}$ satisfies the condition $\forall c \ell^{\prime}\left(D m_{i-1}\left(c \ell^{\prime}\right) \Leftrightarrow\left(\left(C m_{i-1}\left(c \ell^{\prime}\right)\right) \vee O c\left(l t, c \ell^{\prime}\right)\right)\right)$ by construction. If $c \ell^{\prime}$ is any element in $D m_{i-1}$, then it represents some clause $_{1}$ at level $i-1$ such that clause $_{1}$ occurs in the $C N F_{1}$ formula contained in the $\diamond C N F_{1}$ literal represented by $l_{t}$ or $\square$ clause $_{1}$ appears in $T r_{i}$. Hence, all clauses represented in $D m_{i-1}$ are satisfied at $w^{\prime}$ (since $w^{\prime}$ is a successor of $w$ that satisfies $C N F_{1}$ and all literals of the form $\square$ clause represented in $T r_{i}$ are satisfied at $\left.w\right)$. By the induction hypothesis, we conclude that $\xi[i-1]\left(D m_{i-1}\right)$ is true in $\mathcal{S}(\phi)$.

Theorem 3.3. Given a modal CNF formula $\phi$, there is a FPT algorithm that checks if $\phi$ is satisfiable in general models, with treewidth of $\mathcal{S}(\phi)$ and modal depth of $\phi$ as parameters.

Proof. Given $\phi, \mathcal{S}(\phi)$ can be constructed in polynomial time. To check that all clauses of $\phi$ at level md $(\phi)$ are satisfiable in some world $w$ of some Kripke model $\mathcal{M}$, we check whether the formula $\exists C_{\ell_{\operatorname{md}(\phi)}} \forall c_{\ell}\left(C_{\ell_{\operatorname{md}(\phi)}}\left(c_{\ell}\right) \Leftrightarrow\right.$ $\left.\left(C l(c \ell) \wedge L v_{\operatorname{md}(\phi)}(c \ell)\right)\right) \wedge \xi[\operatorname{md}(\phi)]\left(C_{\ell_{\operatorname{md}}(\phi)}\right)$ is true in $\mathcal{S}(\phi)$. By Lemma 3.2, this is possible iff $\phi$ is satisfiable and length of the above formula is linear in $\operatorname{md}(\phi)$. An application of Courcelle's theorem will give us the FPT algorithm.

\subsection{On the relevance of treewidth for modal logic}

Informally, treewidth is a measure of how close a graph is to being a tree. Given a modal logic formula $\phi$, the associated structure $\mathcal{S}(\phi)$ is very similar to the syntax tree of $\phi$. The structure $\mathcal{S}(\phi)$ is not a tree (i.e., it has cycles) because a single propositional variable may be shared by many clauses of the formula. Thus, if very few variables are shared across clauses, $\mathcal{S}(\phi)$ is very close to a tree, i.e., $\mathcal{S}(\phi)$ will have small treewidth. In the example of Fig. 1, if we replace $q$ and $s$ by $r$, the number of shared variables will increase. As can be seen in Fig. 2, the number of cycles will also increase. For example, $e_{1}$ was not part of any cycle in Fig. 1 but forms a cycle with $e_{9}, e_{5}$ and $r$ in Fig. 2 , 


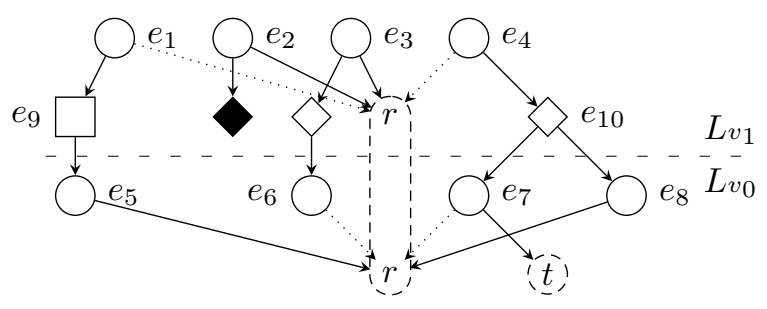

Fig. 2. Relational structure associated with the modal formula $\{\neg r \vee \square[r]\} \wedge\{r \vee \diamond \perp\} \wedge\{r \vee \diamond[\neg r]\} \wedge$ $\{\neg r \vee \diamond[(t \vee \neg r) \wedge(r)]\}$

Treewidth is a very fundamental concept and naturally arises in many contexts, even in industrial applications like software verification [32. Applications of treewidth related techniques to propositional logic is extensively studied - see [12, Section 1.4] and references therein. Modal logic being a natural and very useful extension of propositional logic, we might expect some benefit by exploring applicability of treewidth related techniques to modal logic.

The set of modal formulas with small treewidth is powerful enough to encode complex formulas. In 1, Lemma 1], there is a translation of propositional CNF formulae into equivalent modal formulae. We can verify that the resulting modal formula always has a small constant treewidth (the resulting modal formula uses only one propositional variable). Hence, the restriction of bounded treewidth is not a severe one. Given a formula $\phi, \mathcal{S}(\phi)$ can be computed in PTIME. Though computing treewidth of $\mathcal{S}(\phi)$ is NP-complete, it is FPT when parameterized by treewidth.

\section{Models with Euclidean property}

In this section, we will investigate the parameterized complexity of satisfiability in Euclidean models. The main observation leading to the FPT algorithm is the fact that if a modal formula is satisfied in a Euclidean model, then it is satisfied in a rather simple model. As proved in [22, if a modal formula is satisfied at some world $w_{0}$ in some Euclidean model $\mathcal{M}$, then it is satisfied in a model whose underlying frame is of the form $\left(W \cup\left\{w_{0}\right\}, \mapsto\right)$ where $W \times W \subseteq \mapsto$. Therefore, almost all worlds are successors of almost all other worlds. If one world satisfies a formula $\square$ clause $_{1}$, then almost all worlds satisfy the formula clause $_{1}$ (and hence satisfy $\square$ clause $_{1}$ as well). If one world satisfies a formula $\diamond C N F_{1}$, then almost all worlds satisfy $\diamond C N F_{1}$ as well. Thus, most of the worlds are very similar to each other and we can reason about them using small MSO formulae. This holds even if we add more properties like reflexivity, transitivity etc. The rest of this section is devoted to proving the following theorem.

Theorem 4.1. Let $\phi$ be a modal CNF formula. With treewidth of $\mathcal{S}(\phi)$ as parameter, there is a FPT algorithm for checking whether $\phi$ is satisfiable in a Kripke model that satisfies Euclidean property and any combination of reflexivity, symmetry and transitivity.

We will drop all unary relations $\left(L v_{i}\right)_{0 \leq i \leq \operatorname{md}(\phi)}$. Instead, we will have one unary relation $P v$ containing all domain elements representing propositional variables and one unary relation $H_{l}$ containing all other domain elements. This will not change the treewidth of $\mathcal{S}(\phi)$. To make the presentation easier to follow, we will use informal description of MSO formulae. Let $C_{\ell_{1}}$ and $G C_{\ell}$ be sets of clauses (we will see later that clauses in $G C_{\ell}$ will be satisfied in almost all worlds of a model). The following MSO formula checks if all clauses in $C_{\ell 1}$ are satisfiable in a model in which, all worlds satisfy all clauses in $G C_{\ell}$.

$$
\begin{aligned}
& \chi\left(C_{\ell_{1}}, G C_{\ell}\right) \triangleq \exists T_{r} \subseteq(L t \cap D \diamond): \exists T_{r_{0}} \subseteq P v: \\
& G_{\ell t}=\left\{l_{t} \in(L t \cap B \square) \mid \exists c_{\ell} \in G C_{\ell} \wedge O_{c}\left(l_{t}, c_{\ell}\right)\right\} \\
& \wedge \forall c_{\ell} \in\left(C_{\ell_{1}} \cup G C_{\ell}\right): \\
& \exists l_{t} \in\left(G_{\ell t} \cup T_{r} \cup T_{r_{0}}\right): O_{c}\left(c_{\ell}, l_{t}\right) \\
& \vee \exists l_{t} \in P v \backslash T_{r_{0}}: \overline{O_{c}}\left(c_{\ell}, l_{t}\right) \\
& \wedge \forall l_{t} \in T_{r}: \exists T_{r_{1}} \subseteq P v: \\
& D_{m}=\left\{c \ell^{\prime} \in C l \mid O_{c}\left(l t, c^{\prime}\right)\right\} \Rightarrow \\
& \forall c_{\ell} \in\left(D_{m} \cup G^{\prime} C_{\ell}\right): \\
& \exists l t^{\prime} \in\left(T_{r} \cup G_{\ell t} \cup T_{r_{1}}\right): O_{c}\left(c_{\ell}, l t^{\prime}\right) \\
& \vee \exists l t^{\prime} \in P v \backslash T_{r_{1}}: \overline{O_{c}}\left(c_{\ell}, l t^{\prime}\right)
\end{aligned}
$$


Lemma 4.2. Let $C_{\ell_{1}}$ and $G C_{\ell}$ be sets of clauses occurring in a modal $C N F$ formula $\phi$. If $\chi\left(C_{\ell_{1}}, G C_{\ell}\right)$ is true in $\mathcal{S}(\phi)$, then there is a Kripke model $\mathcal{M}$ and a world $w$ in it such that:

1. $w$ satisfies all clauses in $C_{\ell 1}$,

2. all worlds in $\mathcal{M}$ satisfy all clauses in $G C_{\ell}$,

3. the accessibility relation $\mapsto$ in $\mathcal{M}$ is the equivalence relation on the set of all worlds in $\mathcal{M}$ and

4. if a new world satisfying all clauses in $G C_{\ell}$ is added to $\mathcal{M}$ (making it accessible from all existing worlds of $\mathcal{M}), w$ will still satisfy all clauses in $C_{\ell_{1}}$.

Proof. Suppose $\chi\left(C_{\ell_{1}}, G C_{\ell}\right)$ is true in $\mathcal{S}(\phi)$. We will build a Kripke model $\mathcal{M}$ satisfying the required properties. To begin with, there must be a set $T r$ of literals of the form $\diamond C N F$ and a set $T r_{0}$ of propositional variables such that the rest of the formula $\chi\left(C_{\ell_{1}}, G C_{\ell}\right)$ is true in $\mathcal{S}(\phi)$. Let $G_{\ell t}=\left\{l_{t} \in(L t \cap B \square) \mid \exists c_{\ell} \in G C_{\ell} \wedge O_{c}\left(l_{t}, c_{\ell}\right)\right\}$ be the set of literals of the form $\square$ clause such that the corresponding clause is in $G C_{\ell}$. We will start with one world $w$ in our model in which precisely those propositional variables are set to $T$ that are in the set $T r_{0}$. We will then add exactly one world $w_{i}$ for each literal $l_{t}$ in $T r$. For any literal $l_{t}$ in $T r$, there will be a subset $T r_{1}$ of propositional variables such that last four conditions of $\chi\left(C_{\ell_{1}}, G C_{\ell}\right)$ are true in $\mathcal{S}(\phi)$. In the world $w_{i}$, we will set precisely those propositional variables to $T$ that are in the set $T r_{1}$ corresponding to $l_{t_{i}}$. Our model consists of the above worlds and the accessibility relation $\mapsto$ is the equivalence relation on the set of all worlds. For any literal $l_{t_{i}} \in T r$ (which is of the form $\diamond C N F$ ), let $D m_{i}=\left\{c \ell^{\prime} \in C l \mid O c\left(l_{t_{i}}, c \ell^{\prime}\right)\right\}$ be the set of clauses that make up the corresponding $C N F$ formula. By induction on modal depth of any clause $c_{\ell}$, we will prove that if $c_{\ell} \in C_{\ell} \cup G C_{\ell}$, then $c_{\ell}$ is satisfied in $w$ and if $c_{\ell} \in D m_{i} \cup G C_{\ell}$, then $c_{\ell}$ is satisfied in $w_{i}$.

In the base case, modal depth of $c_{\ell}$ is 0 . We will first prove that if $c_{\ell} \in C_{\ell_{1}} \cup G C_{\ell}$, then $c_{\ell}$ is satisfied in $w$. Suppose $\exists l_{t} \in\left(G_{\ell t} \cup T_{r} \cup T_{r_{0}}\right): O_{c}\left(c_{\ell}, l_{t}\right)$ is true in $\mathcal{S}(\phi)$. If $l_{t} \in G_{\ell t} \cup T_{r}$, then $l_{t}$ will have modal depth at least 1 (because it is of the form $\square$ clause or $\diamond C N F$ ) and hence modal depth of $c_{\ell}$ (which contains $l_{t}$ as a sub-formula) will also be more than 1 . Hence, $l_{t} \in T_{r_{0}}$. This means that $l_{t}$ is a propositional variable set to $\top$ in $w$ that occurs positively in $c_{\ell}$ and hence $c_{\ell}$ is satisfied in $w$. If $\exists l t \in P v \backslash T_{r_{0}}: \overline{O_{c}}\left(c_{\ell}, l_{t}\right)$ is true in $\mathcal{S}(\phi)$, then there is a propositional variable set to $\perp$ in $w$ that occurs negatively in $c_{\ell}$ and hence, $c_{\ell}$ is satisfied in $w$. Now, we will take up the case of $c_{\ell} \in D m_{i} \cup G C_{\ell}$. In the formula $\chi\left(C_{\ell_{1}}, G C_{\ell}\right)$, suppose $\exists l t^{\prime} \in\left(T_{r} \cup G_{\ell t} \cup T_{r_{1}}\right): O c\left(c_{\ell}, l t^{\prime}\right)$ is true in $\mathcal{S}(\phi)$. As before, $l t^{\prime}$ has be to in $T_{r_{1}}$, which means that there is a propositional variable set to $T$ at $w_{i}$ that occurs positively in $c_{\ell}$. Hence, $c_{\ell}$ is satisfied in $w_{i}$. If on the other hand, $\exists l^{\prime} \in P v \backslash T r_{1}: \overline{O_{c}}\left(c_{\ell}, l t^{\prime}\right)$ is true, then there is a propositional variable set to $\perp$ at $w_{i}$ that occurs negatively in $c_{\ell}$. Hence, $c_{\ell}$ is satisfied in $w_{i}$.

For the induction step, suppose $c_{\ell} \in\left(C_{\ell_{1}} \cup G C_{\ell}\right)$. Suppose that in $\mathcal{S}(\phi)$, the formula $\exists l_{t} \in\left(G_{\ell t} \cup T r \cup T_{r_{0}}\right)$ : $O_{c}\left(c_{\ell}, l_{t}\right)$ is true. If $l_{t} \in G_{\ell t}$, then it is of the form $\square$ clause such that the corresponding clause (of modal depth lower than $c_{\ell}$ ) is in $G C_{\ell}$. By the induction hypothesis, all worlds in $\mathcal{M}$ will satisfy all clauses in $G C_{\ell}$ of modal depth less than $c_{\ell}$ (and by condition 4 of the lemma, all new worlds added will also satisfy all clauses in $G C_{\ell}$ ) and hence $\square$ clause is satisfied in $w$ and hence $c_{\ell}$ is satisfied in $w$. If $l_{t} \in T_{r}$, then $l_{t}$ is a literal of the form $\diamond C N F$ such that there is a world $w_{i}$ in $\mathcal{M}$ added to satisfy the corresponding $C N F$ formula. All clauses in this $C N F$ formula (which form the set $D m_{i}$ ) have modal depth less than $c_{\ell}$ and by the induction hypothesis, they are all satisfied at $w_{i}$. Hence, $w_{i}$ satisfies the corresponding $C N F$ formula, and hence $w$ satisfies the corresponding $\diamond C N F$ formula (since $w_{i}$ is a successor of $w$ ) and hence $c_{\ell}$ is satisfied at $w$. If $l_{t} \in T r_{0}$, then $l_{t}$ is a propositional variable set to $T$ in $w$ and that occurs positively in $c_{e}$. Hence, $c e$ is satisfied in $w$. On the other hand, if $\exists l_{t} \in P v \backslash T_{r_{0}}: \overline{O_{c}}\left(c_{e}, l_{t}\right)$ is true in $\mathcal{S}(\phi)$, then $l_{t}$ is a propositional variable set to $\perp$ in $w$ and that occurs negatively in $c$. Hence, in this case also, $c_{\ell}$ is satisfied in $w$.

Finally, for the induction step, suppose $c_{\ell} \in D m_{i} \cup G C_{\ell}$. Suppose that in $\mathcal{S}(\phi)$, the formula $\exists l t^{\prime} \in$ $\left(T_{r} \cup G_{\ell t} \cup T_{r_{1}}\right): O_{c}\left(c_{\ell}, l_{t}^{\prime}\right)$ is true. If $l_{t}^{\prime} \in G_{\ell t}$, then it is of the form $\square$ clause such that the corresponding clause (of modal depth lower than $c_{\ell}$ ) is in $G C_{\ell}$. By the induction hypothesis, all worlds in $\mathcal{M}$ will satisfy all clauses in $G C_{\ell}$ of modal depth less than $c_{\ell}$ (and by condition 4 of the lemma, all new worlds added will also satisfy all clauses in $G C_{\ell}$ ) and hence $\square$ clause is satisfied in $w_{i}$ and hence $c_{\ell}$ is satisfied in $w_{i}$. If $l t^{\prime} \in T r$, then $l t^{\prime}$ is a literal of the form $\diamond C N F$ such that there is a world $w_{i^{\prime}}$ in $\mathcal{M}$ added to satisfy the corresponding $C N F$ formula. All clauses in this $C N F$ formula (which form the set $D m_{i^{\prime}}$ ) have modal depth less than $c_{\ell}$ and by the induction hypothesis, they are all satisfied at $w_{i^{\prime}}$. Hence, $w_{i^{\prime}}$ satisfies the corresponding $C N F$ formula, and hence $w_{i}$ satisfies the corresponding $\triangle C N F$ formula (since $w_{i^{\prime}}$ is a successor of $w_{i}$ ) and hence $c_{\ell}$ is satisfied at $w_{i}$. If $l t^{\prime} \in T r_{1}$, then $l t^{\prime}$ is a propositional variable set to $T$ in $w_{i}$ and that occurs positively in $c_{\ell}$. Hence, $c_{\ell}$ is satisfied in $w_{i}$. On the other hand, if $\exists l t^{\prime} \in P v \backslash T r_{1}: \overline{O c}\left(c_{\ell}, l t^{\prime}\right)$ is true in $\mathcal{S}(\phi)$, then $l t^{\prime}$ is a propositional variable set to $\perp$ in $w_{i}$ and that occurs negatively in $c_{\ell}$. Hence, in this case also, $c_{\ell}$ is satisfied in $w_{i}$. This completes the induction step and hence the proof. 
The following formula makes use of $\chi\left(C_{\ell_{1}}, G C_{\ell}\right)$ to check if a set of clauses $C_{\ell_{0}}$ is satisfiable in an Euclidean model.

$$
\begin{aligned}
& \chi\left(C_{\ell_{0}}\right) \triangleq \exists T_{0} \subseteq L t: \forall l_{t} \in T_{r_{0}}: \exists c_{\ell} \in C_{\ell_{0}}: O_{c}\left(c_{\ell}, l_{t}\right) \\
& \wedge \forall c_{\ell} \in C_{\ell_{0}}: \\
& \exists l t \in T_{r_{0}}: O_{c}\left(c_{\ell}, l_{t}\right) \\
& \vee \exists l t \in P v \backslash T r_{0}: \overline{O c}\left(c_{\ell}, l_{t}\right) \\
& \wedge C_{m_{0}}=\left\{c_{\ell} \in C_{l} \mid \exists l t \in\left(T_{r_{0}} \cap B \square\right) \wedge O_{c}\left(l_{t}, c_{\ell}\right)\right\} \Rightarrow \\
& \exists G C_{\ell} \subseteq(C l): \forall c_{\ell} \in G C_{\ell}: \exists l t \in(L t \cap B \square): O_{c}\left(l_{t}, c_{\ell}\right) \\
& \wedge \forall l_{t} \in\left(T_{r_{0}} \cap D \diamond\right): \\
& \quad D_{m_{0}}=\left\{c_{\ell} \in C_{l} \mid O_{c}\left(l_{t}, c_{\ell}\right)\right\} \Rightarrow \\
& \chi\left(D m_{0} \cup C_{m_{0}}, G C_{\ell}\right)
\end{aligned}
$$

Lemma 4.3. Let $C_{\ell_{0}}$ be a set of clauses occurring in a modal $C N F$ formula $\phi . C N F\left(\mathrm{C}_{\ell_{0}}\right)$ is satisfiable at a world $w$ in an Euclidean model $\mathcal{M}$ in which $w$ is not its own successor iff $\chi\left(C_{\ell_{0}}\right)$ is true in $\mathcal{S}(\phi)$.

Proof. Suppose $\chi\left(C_{\ell_{0}}\right)$ is true in $\mathcal{S}(\phi)$. We will build an Euclidean Kripke model $\mathcal{M}$ satisfying $C N F\left(\mathrm{C}_{\ell_{0}}\right)$. We will begin with a single world $w$. In $w$, precisely those propositional variables are set to $T$ that appear in the set $T r_{0}$ that witnesses truth of $\chi\left(C_{\ell_{0}}\right)$ in $\mathcal{S}(\phi)$. For now, we will assume that literals of the form $\triangleright C N F$ and $\square$ clause in $T_{r_{0}}$ will be satisfied in $w$ by addition of suitable worlds. If $c_{\ell}$ is any clause in $C_{\ell_{0}}$, then either $\exists l t \in T_{r_{0}}: O_{c}\left(c \ell, l_{t}\right)$ or $\exists l t \in P v \backslash T_{r_{0}}: \overline{O_{c}}\left(c_{\ell}, l_{t}\right)$ is true. In the former case, a propositional variable that is set to $T$ at $w$ or a literal of the form $\triangle C N F$ or $\square$ clause that is satisfied in $w$ occurs in $c_{\ell}$ and hence $c_{\ell}$ is satisfied in $w$. In the later case, a propositional variable set to $\perp$ in $w$ occurs negatively in $c_{\ell}$ and hence $c_{\ell}$ is satisfied in $w$.

As promised, we will now add suitable successors such that all literals of the form $\diamond C N F$ and $\square$ clause in $T_{r_{0}}$ are satisfied at $w$. Let $C_{m_{0}}=\left\{c_{\ell} \in C l \mid \exists l t \in\left(T_{r_{0}} \cap B \square\right) \wedge O_{c}\left(l_{t}, c_{\ell}\right)\right\}$ be the set of clauses we have committed to satisfy in all successors of $w$ by choosing the corresponding $\square$ clause to be in $T r_{0}$. For any literal $l_{t}$ of the form $\diamond C N F$ in $T r_{0} \cap D \diamond$, let $D_{m_{0}}=\left\{c_{\ell} \in C l \mid O_{c}\left(l_{t}, c_{\ell}\right)\right\}$ be the set of clauses in the corresponding $C N F$ formula. Since $\chi\left(D m_{0} \cup C_{m_{0}}, G C_{\ell}\right)$ is true in $\mathcal{S}(\phi)$, there is a model $\mathcal{M}_{1}$ and a world $w_{1}$ in it as specified in Lemma 4.2, such that $w_{1}$ satisfies all clauses in $D_{m_{0}} \cup C_{m_{0}}$ and all worlds in $\mathcal{M}_{1}$ satisfy all clauses in $G C_{\ell}$. Let $\left(\mathcal{M}_{1}, w_{1}\right),\left(\mathcal{M}_{2}, w_{2}\right), \ldots$ be the models given by Lemma 4.2 for the demand sets created by each of the literals of the form $\diamond C N F$ in $T r_{0} \cap D \diamond$. Adding all worlds of $\mathcal{M}_{1}, \mathcal{M}_{2}, \ldots$ to $\mathcal{M}$ and making $w_{1}, w_{2}, \ldots$ successors of $w$ will result in all literals of the form $\diamond C N F$ in $T r_{0} \cap D \diamond$ being satisfied at $w$ in $\mathcal{M}$. Since $w_{1}, w_{2}, \ldots$ all satisfy all clauses in $C_{m_{0}}$, all literals of the form $\square$ clause in $T r_{0} \cap B \square$ are also satisfied at $w$ in $\mathcal{M}$. Making all worlds other than $w$ successors of all worlds other than $w$ will ensure that $\mathcal{M}$ is based on an Euclidean frame. Condition 4 of Lemma 4.2 will ensure that due to the additional accessibility relation pairs created, the worlds $w_{1}, w_{2}, \ldots$ will not stop satisfying clauses required to satisfy the demands created by literals in $T_{r_{0}}$.

Now, suppose that $C N F\left(\mathrm{C}_{\ell_{0}}\right)$ is satisfied in an Euclidean model. We will prove that $\chi\left(C_{\ell_{0}}\right)$ is true in $\mathcal{S}(\phi)$. As proved in 22], $C N F\left(\mathrm{C}_{\ell_{0}}\right)$ is satisfied in a model $\mathcal{M}$ at a world $w$ such that the underlying frame of $\mathcal{M}$ is of the form $(W \cup\{w\}, \mapsto)$ such that $W \times W \subseteq \mapsto$. As stated in the lemma, $w$ is not its own successor. If $w$ was the successor of any other world, then Euclidean property will force $w$ to be its own successor, hence $w$ can not be the successor of any other world. To prove that $\chi\left(C_{\ell_{0}}\right)$ is true in $\mathcal{S}(\phi)$, we will first construct a set $T_{r_{0}}$ of literals. Since $C N F\left(\mathrm{C}_{\ell_{0}}\right)$ is satisfied at $w$, for every clause in $C_{\ell_{0}}$, there must be a literal occurring in that clause satisfied at $w$. Let $T r_{0}$ be the set of such literals of the form $\square$ clause or $\diamond C N F$ and the propositional variables set to $T$ in $w$ and occurring positively in some clause in $C_{\ell_{0}}$. The condition $\forall l_{t} \in T_{r_{0}}: \exists c_{\ell} \in C_{\ell_{0}}: O_{c}\left(c_{\ell}, l_{t}\right)$ is true by construction of $T_{r_{0}}$. If $c_{\ell}$ is any clause in $C_{\ell_{0}}$, then either

1. there is some literal of the form $\square$ clause or $\diamond C N F$ or a positively occurring propositional variable that occurs in $c_{\ell}$ and present in $T_{r_{0}}$ (in which case $\exists l t \in T_{r_{0}}: O c\left(c \ell, l_{t}\right)$ is true) or

2. there is a negatively occurring propositional variable that is set to $\perp$ in $w$ (in which case $\exists l_{t} \in P v \backslash T_{r_{0}}$ : $\overline{O_{c}}\left(c_{\ell}, l_{t}\right)$ is true).

Let $C_{m_{0}}=\left\{c_{\ell} \in C_{l} \mid \exists l t \in\left(T_{r_{0}} \cap B \square\right) \wedge O_{c}\left(l_{t}, c_{\ell}\right)\right\}$ be the set of clauses that are satisfied in all successors of $w$. Let $G C_{\ell}=\left\{c_{\ell} \in C_{l} \mid \exists l_{t} \in\left(L_{t} \cap B \square\right) \wedge O_{c}\left(l_{t}, c_{\ell}\right) \wedge \mathcal{M}, w^{\prime} \models l t, w^{\prime} \neq w\right\}$ be the set of all clauses such that corresponding $\square$ clause formula is satisfied at some world $w^{\prime}$ other than $w$. Since all worlds other than $w$ are successors of $w^{\prime}$, all worlds other than $w$ satisfy all clauses in $G C_{\ell}$. The condition $\forall c_{\ell} \in G C_{\ell}: \exists l_{t} \in(L t \cap B \square): O_{c}\left(l_{t}, c_{\ell}\right)$ is true by construction of $G C_{\ell}$. Note that $G C_{\ell}$ and $T_{r_{0}} \cap D \diamond$ will 
be empty if $w$ has no successors, hence the rest of $\chi\left(C \ell_{0}\right)$ is vacuously true. For any literal $l_{t} \in \operatorname{Tr}_{0} \cap D \diamond$, let $D m_{0}=\left\{c_{\ell} \in C l \mid O_{c}\left(l t, c_{\ell}\right)\right\}$ be the set of clauses in the $C N F$ formula contained in $l t$. In $\mathcal{M}$, there is a successor $w_{1}$ of $w$ that satisfies all clauses in $D m_{0} \cup C m_{0}$. We will prove that $\chi\left(D m_{0} \cup C m_{0}, G C_{\ell}\right)$ is true in $\mathcal{S}(\phi)$.

We first select a subset $T_{r} \subseteq(L t \cap D \diamond)$ so that the rest of the formula $\chi\left(D m_{0} \cup C_{m_{0}}, G C_{\ell}\right)$ can be satisfied. Let $T r=\left\{l_{t} \in(L t \cap D \diamond) \mid \mathcal{M}, w^{\prime} \models l t, w^{\prime} \neq w\right\}$ be the set of literals of the form $\diamond C N F$ such that some world $w^{\prime}$ other than $w$ satisfies the $\searrow C N F$ formula (since $w$ is not a successor of $w^{\prime}$, some other world $w^{\prime \prime}$ succeeding $w^{\prime}$ will satisfy the corresponding $C N F$ formula). Let $T r_{0}$ be the set of propositional variables set to $T$ in the world $w_{1}$ mentioned above. Let $G_{\ell t}=\left\{l_{t} \in\left(L_{t} \cap B \square\right) \mid \exists c_{\ell} \in G C_{\ell} \wedge O_{c}\left(l_{t}, c_{\ell}\right)\right\}$ be the set of literals of the form $\square$ clause such that the corresponding clause is in $G C_{\ell}$. Let $c_{\ell}$ be any clause in $D m_{0} \cup C_{m_{0}} \cup G C_{\ell}$. Since $w_{1}$ satisfies $c_{\ell}$, there must be a literal $l_{t}$ occurring in $c_{\ell}$ such that $l_{t}$ is satisfied in $w_{1}$.

1. If $l_{t}$ is of the form $\square$ clause, then it is in $G_{\ell t}$ and hence $\exists l_{t} \in G_{\ell t}: O_{c}\left(c_{\ell}, l_{t}\right)$ is true.

2. If $l_{t}$ is of the form $\diamond C N F$, then it is in $T_{r}$ and hence $\exists l_{t} \in T_{r}: O_{c}\left(c_{e}, l_{t}\right)$ is true.

3. If $l_{t}$ is a positively occurring propositional variable, then $\exists l_{t} \in T_{r_{0}}: O_{c}\left(c_{\ell}, l_{t}\right)$ is true.

4. If $l_{t}$ is a negatively occurring propositional variable, then $\exists l_{t} \in P v \backslash T r_{0}: \overline{O_{c}}\left(c_{\ell}, l_{t}\right)$ is true.

Let $l_{t} \in T_{r}$ be any literal of the form $\diamond C N F$ in $T r$. By definition of $T_{r}$, there is some world $w^{\prime}$ other than $w$ such that $w^{\prime}$ satisfies the corresponding $C N F$ formula. Let $T_{r_{1}}$ be the set of propositional variables set to $T$ in $w^{\prime}$ and let $D_{m}=\left\{c^{\prime} \in C l \mid O_{c}\left(l t, c \ell^{\prime}\right)\right\}$ be the set of clauses in the $C N F$ formula in $l t$. Let $c e$ be any clause in $D m \cup G C$ e. Since $w^{\prime}$ satisfies $c_{\ell}$, there must be a literal $l t^{\prime}$ occurring in $c_{\ell}$ such that $l t^{\prime}$ is satisfied in $w^{\prime}$.

1. If $l t^{\prime}$ is of the form $\square$ clause, then it is in $G_{\ell t}$ and hence $\exists l t^{\prime} \in G_{\ell t}: O_{c}\left(c_{\ell}, l_{t}\right)$ is true.

2. If $l t^{\prime}$ is of the form $\diamond C N F$, then it is in $T r$ and hence $\exists l t^{\prime} \in T_{r}: O_{c}\left(c_{\ell}, l_{t}^{\prime}\right)$ is true.

3. If $l t^{\prime}$ is a positively occurring propositional variable, then $\exists l t^{\prime} \in T_{r_{1}}: O c\left(c e, l t^{\prime}\right)$ is true.

4. If $l t^{\prime}$ is a negatively occurring propositional variable, then $\exists l^{\prime} \in P v \backslash \operatorname{Tr}_{1}: \overline{O_{c}}\left(c_{\ell}, l t^{\prime}\right)$ is true.

Suppose a modal formula is satisfied at a world $w$ in an Euclidean model where $w$ is its own successor. Then Euclidean property will force the accessibility relation $\mapsto$ to be the equivalence relation on the set of all worlds. Hence, any $\square$ clause literal chosen to be satisfied in $w$ will result in all worlds (including $w$ ) satisfying the corresponding clause. This can be easily handled by modifying $\chi\left(C_{\ell_{0}}\right)$ as follows.

$$
\begin{aligned}
\chi^{\prime}\left(C_{\ell_{0}}\right) \triangleq \exists T r_{0} \subseteq L_{t} & : \\
\exists G C_{\ell} \subseteq(C l): \forall c_{\ell} \in G C_{\ell}: \exists l t \in(L t \cap B \square): O_{c}\left(l_{t}, c_{\ell}\right) & \wedge \forall c_{\ell} \in\left(C_{\ell_{0}} \cup G C_{\ell}\right): \\
& \exists l t \in T r_{0}: O c\left(c_{\ell}, l_{t}\right) \\
& \vee \exists l_{t} \in P v \backslash T r_{0}: \overline{O c}\left(c_{\ell}, l_{t}\right) \\
\wedge \forall l_{t} \in & \left(T_{r_{0}} \cap D \diamond\right): \\
& D_{m_{0}}=\left\{c \ell \in C l \mid O_{c}\left(l_{t}, c_{\ell}\right)\right\} \Rightarrow \\
& \chi\left(D m_{0}, G C_{\ell}\right)
\end{aligned}
$$

Now to check if a modal formula $\phi$ is satisfiable in an Euclidean model, we just have to check if $\chi\left(C_{\ell_{0}}\right) \vee \chi^{\prime}\left(C_{\ell_{0}}\right)$ is true in $\mathcal{S}(\phi)$, where $C_{\ell_{0}}$ is the set of clauses at the highest level. An application of Courcelle's theorem will give us the FPT algorithm. Note that in this case, the size of the MSO formula we need to check is independent of modal depth.

To check if a modal formula $\phi$ is satisfiable in a reflexive and Euclidean model, we just check if $\chi^{\prime}\left(C_{\ell_{0}}\right)$ is true in $\mathcal{S}(\phi)$.

Suppose a modal formula $\phi$ is satisfied at some world $w$ in an Euclidean and symmetric model. If $w$ has any other successors, then Euclidean property will force all worlds reachable from $w$ to be successors of $w$ and $w$ to be a successor of all worlds reachable from $w$. This is same as a reflexive and Euclidean model and can be handled by $\chi^{\prime}\left(C_{\ell_{0}}\right)$. If $w$ has no other successors but is its own successor it can again be handled by $\chi^{\prime}\left(C_{\ell_{0}}\right)$. If $w$ has no successors and is not its own successor, then all clauses of $\phi$ at the highest level are satisfied at $w$ by literals of the form $\square$ clause or propositional variables. This can be easily checked by a small MSO formula.

\subsection{Euclidean and transitive models}

Suppose we want to check satisfiability of a modal CNF formula in models that are both Euclidean and transitive. As seen above, the modal CNF formula is satisfied in a model with an underlying frame of the 
form $(W \cup\{w\}, \mapsto)$ where $W \times W \subseteq \mapsto$. In addition, all other worlds are successors of $w$. Hence any literal of the form $\square$ clause satisfied at $w$ will result in all other worlds satisfying the corresponding clause. This can be handled by modifying $\chi\left(C_{\ell}\right)$ as follows.

$$
\begin{aligned}
& \chi^{\prime \prime}\left(C_{\ell_{0}}\right) \triangleq \exists T_{r_{0}} \subseteq L t: \forall l_{t} \in T_{r_{0}}: \exists c_{\ell} \in C_{\ell_{0}}: O_{c}\left(c_{\ell}, l_{t}\right) \\
& \wedge \forall c_{\ell} \in C_{\ell_{0}}: \\
& \exists l t \in T_{r_{0}}: O_{c}\left(c_{\ell}, l_{t}\right) \\
& \vee \exists l t \in P v \backslash T r_{0}: \overline{O c}\left(c_{\ell}, l_{t}\right) \\
& \wedge C_{0}=\left\{c \ell \in C l \mid \exists l t \in\left(T_{r_{0}} \cap B \square\right) \wedge O_{c}\left(l_{t}, c_{\ell}\right)\right\} \Rightarrow \\
& \exists G C_{\ell} \subseteq(C l): \forall c_{\ell} \in G C_{\ell}: \exists l t \in(L t \cap B \square): O_{c}\left(l_{t}, c_{\ell}\right) \\
& \wedge \forall l_{t} \in\left(T_{r_{0}} \cap D \diamond\right): \\
& D m_{0}=\left\{c_{\ell} \in C_{l} \mid O_{c}\left(l_{t}, c_{\ell}\right)\right\} \Rightarrow \\
& \chi\left(D m_{0} \cup C_{m_{0}}, G C_{\ell} \cup C_{m_{0}}\right)
\end{aligned}
$$

The Euclidean property is very strong in the sense that it makes the complexity of infinitely many modal logics drop from PSPACE-hard to NP-complete [18. One might hope for extending the results of this section to any modal logic whose frames is a subset of Euclidean frames. The results in [18 use semantic characterizations while our MSO formulae can only reason about syntax of modal logic formulae. Even though there is a close relation between the syntax and semantics of modal logic of Euclidean frames (which have been used to obtain the results of this section), it seems difficult to exploit this relation to obtain FPT algorithms for arbitrary extensions of modal logic of Euclidean frames. It remains to be seen if other tools from the theory of MSO logic on graphs can be used to achieve this.

\section{Reflexive models}

As an example of how the basic technique described in section 3 can be extended to satisfiability in models satisfying some other properties, we will show satisfiability in reflexive models. We will need the following MSO formula to define the set of vertices reachable from a given vertex in a finite directed acyclic graph.

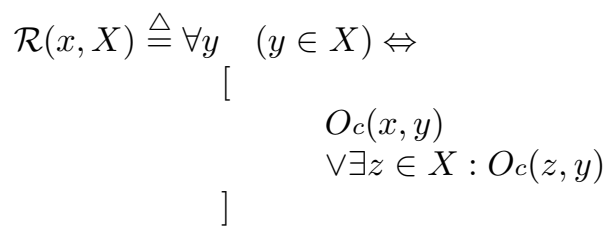

Lemma 5.1. Let $G$ be a finite directed acyclic graph in which, $O_{c}$ is the binary relation represented by the directed edges. Let $x$ be a vertex and $X$ be a subset of vertices in $G$. Then, $X$ is the set of precisely those vertices reachable from $x$ by a directed path of length 1 or more iff $\mathcal{R}(x, X)$ defined in (9) is true in $G$.

Proof. Suppose $X$ is the set of precisely those vertices reachable from $x$ by a directed path of length 1 or more. If some vertex $y$ is in $X$ (i.e., there is a directed path of length 1 or more from $x$ to $y$ ), we will prove that $O c(x, y) \vee \exists z \in X: O c(z, y)$ is true in $G$. If the length of the path from $x$ to $y$ is 1 , there is an edge from $x$ to $y$, making $O_{c}(x, y)$ true in $G$. If the directed path between $x$ and $y$ is at least 2 and is of the form $x \rightarrow y^{\prime} \rightarrow \cdots \rightarrow y^{\prime \prime} \rightarrow y$, then we can take $y^{\prime \prime}$ as witness for $z$ in $\exists z \in X: O c(z, y)$, making $\exists z \in X: O c(z, y)$ true in $G$. On the other hand, suppose $O_{c}(x, y) \vee \exists z \in X: O_{c}(z, y)$ is true in $G$ for some vertex $y$. We will prove that $y$ is in $X$ (i.e., there is a directed path of length 1 or more from $x$ to $y$ ). Suppose $O c(x, y)$ is true in $G$. Then there is an edge from $x$ to $y$, which is a directed path of length 1. Suppose $\exists z \in X: O c(z, y)$ is true $G$, then there is a directed path of length 1 or more from $x$ to $z$ (since $z$ is in $X$ ). Appending the edge from $z$ to $y$ to this path gives us a path of length 2 or more from $x$ to $y$.

Now, suppose that $\forall y \quad(y \in X) \Leftrightarrow\left[O_{c}(x, y) \vee\left(\exists z \in X: O_{c}(z, y)\right)\right]$ is true in $G$. We will prove that $X$ is the set of precisely those vertices reachable from $x$ by a directed path of length 1 or more. We will first prove that $X$ does not contain any vertex not reachable from $x$. Suppose to the contrary that there is a vertex $y$ in $X$ not reachable from $x$. Since $y \in X, O_{c}(x, y) \vee\left(\exists z \in X: O_{c}(z, y)\right)$ is true in $G$. Since $O_{c}(x, y)$ cannot be true (as that would mean $y$ is reachable from $x$ ), there is some $z_{1} \in X$ with $O_{c}\left(z_{1}, y\right)$. Since $z_{1}$ is in $X, O_{c}\left(x, z_{1}\right) \vee\left(\exists z \in X: O_{c}\left(z, z_{1}\right)\right)$ is true. Since $O_{c}\left(x, z_{1}\right)$ cannot be true (as that would mean $y$ is reachable from $x)$, there is some $z_{2} \in X$ with $O_{c}\left(z_{2}, z_{1}\right)$. The vertex $z_{2}$ has to be distinct from $y$ and $z_{1}$ since 
otherwise, the fact that $G$ is devoid of directed cycles is violated. Continuing this type of reasoning leads us to an infinite sequence $y, z_{1}, z_{2}, \ldots$ of distinct vertices, contradicting the fact that $G$ is a finite graph. Hence, $X$ does not contain any vertex not reachable from $x$. Next we will prove that every vertex $y$ reachable from $x$ is in $X$, by induction on the length $i$ of the shortest directed path from $x$ to $y$. In the base case $i=1$, there is an edge from $x$ to $y$, which means that $O_{c}(x, y)$ is true in $G$, and $\mathcal{R}(x, X)$ forces $y$ to be in $X$. Suppose there is a directed path of length $i+1$ from $x$ to $y$. Let $z$ be the vertex preceding $y$ in this path. Since there is a directed path of length at most $i$ from $x$ to $z$, we can use induction hypothesis conclude that $z \in X$. Since there is an edge from $z$ to $y, \exists z \in X: O c(z, y)$ is true in $G$, and again $\mathcal{R}(x, X)$ forces $y$ to be in $X$.

Let $C_{\ell_{i}}$ be some set of domain elements representing clauses at level at most $i$. The property $\zeta[i]\left(C_{\ell_{i}}\right)$ defined below checks if there is a reflexive Kripke model $\mathcal{M}$ and a world $w$ in it that satisfies all clauses in $C_{\ell_{i}}$.

$$
\begin{aligned}
& \zeta[0]\left(C_{\ell_{0}}\right) \triangleq \exists T r_{0} \subseteq\left(L t \cap L v_{0}\right): \forall c_{\ell} \in C_{\ell_{0}}: \\
& {\left[\left(\exists l t \in T_{r_{0}}: O c\left(c_{\ell}, l_{t}\right)\right) \vee\left(\exists l t \in\left(L t \cap L v_{0}\right) \backslash T_{r_{0}}: \overline{O_{c}}\left(c_{\ell}, l_{t}\right)\right)\right]} \\
& \zeta[i]\left(C_{\ell_{i}}\right) \triangleq \exists T r_{i} \subseteq L t: \\
& \forall l_{t} \in T_{r_{i}} \quad \exists c_{\ell} \in C_{\ell_{i}}: \exists X:\left(\mathcal{R}\left(c_{\ell}, X\right) \wedge l_{t} \in X\right) \\
& \wedge C m_{i-1}=\left\{c e^{\prime} \in C l \mid \exists l t^{\prime} \in T r_{i} \cap B \square, O c\left(l t^{\prime}, c \ell^{\prime}\right)\right\} \Rightarrow \\
& \forall c_{\ell} \in C_{\ell_{i}} \cup C_{m_{i-1}} \text { : } \\
& {\left[\left(\exists l_{t} \in T_{r_{i}}: O_{c}\left(c_{\ell}, l_{t}\right)\right) \vee\left(\exists l t \in L t \backslash T_{r_{i}}: \overline{O_{c}}\left(c_{\ell}, l_{t}\right)\right)\right]} \\
& \wedge \forall l_{t} \in T_{r_{i}} \cap D \diamond: D m_{i-1}=\left\{c_{\ell} \in C_{l} \mid O_{c}\left(l_{t}, c_{\ell}\right)\right\} \Rightarrow \\
& \zeta[i-1]\left(D m_{i-1} \cup C m_{i-1}\right)
\end{aligned}
$$

Lemma 5.2. The property $\zeta[i]\left(C_{\ell_{i}}\right)$ can be written in a MSO logic formula of size linear in $i$. If $\phi$ is any modal formula in $C N F$ and $C_{\ell_{i}}$ is any subset of domain elements representing clauses at level at most $i$, then $\operatorname{CNF}\left(\mathrm{C}_{\ell_{\mathrm{i}}}\right)$ is satisfiable in a reflexive model iff $\zeta[i]\left(C_{\ell_{i}}\right)$ is true in $\mathcal{S}(\phi)$.

Proof. We will prove the first claim by induction on $i$. We will prove that the length $|\zeta[i]|$ of $\zeta[i]$ is linear in $i$. Let $c$ be the length of $\zeta[i]$ without the length of $\zeta[i-1]$ counted. As can be seen, $|\zeta[0]| \leq c$. Inductively assume that $|\zeta[i-1]| \leq i c$. Then, $|\zeta[i]|=c+|\zeta[i-1]|$. Hence, $|\zeta[i]| \leq c+i c=c(i+1)$.

We will now prove the second claim by induction on $i$.

Base case $i=0$ : Suppose $\zeta[0]\left(C_{\ell_{0}}\right)$ is true in $\mathcal{S}(\phi)$. Hence, there is a subset $T_{r_{0}}$ of domain elements that satisfy all the conditions of $\zeta[0]$ defined in (10). Since all domain elements in $T r_{0}$ represent literals at level 0 and the only literals at level 0 are propositional variables or their negations, $T r_{0}$ is in fact a subset of propositional variables. Consider the reflexive Kripke model $\mathcal{M}$ with a single world $w$ at which, all propositional variables in $T_{r_{0}}$ are set to $T$ and all others are set to $\perp$. We will now prove that all clauses represented in $C_{\ell_{0}}$ are satisfied in $w$. Let $c_{\ell}$ be some element in $C_{\ell_{0}}$ representing some clause. We have that either $\exists l_{t} \in T_{r_{0}}: O_{c}\left(c_{\ell}, l_{t}\right)$ or $\exists l_{t} \in\left(L t \cap L v_{0}\right) \backslash T_{r_{0}}: \overline{O_{c}}\left(c_{\ell}, l_{t}\right)$ is true in $\mathcal{S}(\phi)$. In the first case, a positively occurring propositional variable is set to $T$ in $w$ and in the second case, a negatively occurring propositional variable is set to $\perp$ in $w$.

Now suppose that there is a reflexive Kripke model $\mathcal{M}$ and a world $w$ such that $\mathcal{M}, w \models C N F\left(\mathrm{C}_{\ell_{0}}\right)$. We will prove that $\zeta[0]\left(C_{\ell_{0}}\right)$ is true in $\mathcal{S}(\phi)$. The first requirement is to find a suitable subset $\operatorname{Tr}_{0}$ of domain elements. We will set $T_{r_{0}}$ to be the set of precisely those domain elements that represent propositional variables occurring at level 0 and set to $\top$ in the world $w$. Since every clause $c_{\ell}$ in $C_{\ell_{0}}$ is satisfied in $w$, either there is a positively occurring propositional variable set to $T$ in $w$ or there is a negatively occurring propositional variable set to $\perp$ in $w$. In the first case $\exists l t \in T_{r_{0}}: O_{c}\left(c_{\ell}, l_{t}\right)$ is true and in the second case $\exists l_{t} \in\left(L t \cap L v_{0}\right) \backslash T_{r_{0}}: \overline{O_{c}}\left(c_{\ell}, l_{t}\right)$ is true in $\mathcal{S}(\phi)$. This completes the base case.

Induction step: Suppose $C_{\ell_{i}}$ is a subset of domain elements representing clauses occurring at level at most $i$ and $\zeta[i]\left(C_{\ell_{i}}\right)$ is true in $\mathcal{S}(\phi)$. We will build a reflexive Kripke model $\mathcal{M}$ and prove that it has a world $w$ such that $\mathcal{M}, w \models C N F\left(\mathrm{C}_{\ell_{\mathrm{i}}}\right)$. We will start with a single world $w$. Since $\zeta[i]\left(C_{\ell_{i}}\right)$ is true in $\mathcal{S}(\phi)$, there must be a subset $T r_{i}$ of domain elements satisfying all the conditions of $\zeta[i]\left(C_{\ell_{i}}\right)$. Since $\forall l t \in T_{r_{i}} \quad \exists c_{\ell} \in$ $C_{\ell_{i}}: \exists X:\left(\mathcal{R}\left(c_{\ell}, X\right) \wedge l_{t} \in X\right)$ is true in $\mathcal{S}(\phi)$, all literals in $T_{r_{i}}$ are reachable from some clause in $C_{\ell_{i}}$ in $\mathcal{S}(\phi)$. Hence, all literals in $T r_{i}$ are at level at most $i$. Let $C m_{i-1}=\left\{c e^{\prime} \in C l \mid \exists l t^{\prime} \in T r_{i} \cap B \square, O c\left(l t^{\prime}, c e^{\prime}\right)\right\}$ be the set of clauses that we are committed to satisfy in all successors of $w$ (that includes $w$ as well) as a result of chosing the corresponding $\square$ clause to be in $T_{r_{i}}$. All clauses in $C_{m_{i-1}}$ are at level at most $i-1$. For each literal $l_{t_{1}}$ of the form $\diamond C N F$ in $T_{r_{i}}$, let $D_{m_{i-1}}=\left\{c_{\ell} \in C l \mid O_{c}\left(l_{t_{1}}, c_{\ell}\right)\right\}$ be the set of clauses occurring in $l_{t_{1}}$. 
Since all clauses in $D m_{i-1} \cup C m_{i-1}$ are at level at most $i-1$ and $\zeta[i-1]\left(D m_{i-1} \cup C m_{i-1}\right)$ is true in $\mathcal{S}(\phi)$, we can apply induction hypothesis to conclude that there is a reflexive Kripke model $\mathcal{M}_{1}$ and a world $w_{1}$ in it that satisfies all clauses in $D_{m_{i-1}} \cup C_{m_{i-1}}$. Add all such models $\mathcal{M}_{1}, \mathcal{M}_{2}, \ldots$ to our Kripke model $\mathcal{M}$ we are constructing and make the worlds $w_{1}, w_{2}, \ldots$ successors of $w$. In $w$, set precisely those propositional variables to $T$ that occur in $T r_{i}$. Let $c_{\ell}$ be any clause in $C_{\ell_{i}} \cup C_{m_{i-1}}$. Now, we will prove by induction on modal depth of $c_{\ell}$ that in $\mathcal{M}$, the world $w$ satisfies $c_{\ell}$. If $\exists l_{t} \in L t \backslash T_{r}: \overline{O_{c}}\left(c_{\ell}, l_{t}\right)$ is true, then a propositional variable not in $T r_{i}$ occurs negatively in $c_{\ell}$. Since this propositional variable is set to $\perp$ in $w, c_{\ell}$ is satisfied at $w$. If $\exists l_{t} \in T_{r_{i}}: O c\left(c_{e}, l_{t}\right)$ is true and $l_{t}$ is a propositional variable, then it is set to $\top$ in $w$ and occurs positively in $c_{\ell}$. If $\exists l_{t} \in T_{r_{i}}: O_{c}\left(c_{\ell}, l_{t}\right)$ is true and $l_{t}$ is of the form $\square$ clause, then the corresponding clause is in $C_{m_{i-1}}$ and hence true in all successors of $w$ (including $w$ itself, by induction on modal depth of $c_{\ell}$ ). If $\exists l_{t} \in T_{r_{i}}: O_{c}\left(c_{\ell}, l_{t}\right)$ is true and $l_{t}$ is of the form $\diamond C N F$, then we would have added a world to satisfy the corresponding $C N F$ formula.

Now we will prove the other direction of the induction step. Suppose $C_{\ell_{i}}$ is a subset of domain elements representing clauses occurring at level at most $i$ and that there is a reflexive Kripke model $\mathcal{M}$ and a world $w$ such that $\mathcal{M}, w \models C N F\left(\mathrm{C}_{\ell_{\mathrm{i}}}\right)$. We will prove that $\zeta[i]\left(C_{\ell_{i}}\right)$ is true in $\mathcal{S}(\phi)$. To begin with, we will choose $T r_{i}$ to be the set of precisely those literals occurring at level $i$ or below that are satisfied at $w$ and occur as subformulas of some clause in $C_{\ell_{i}}$. This will ensure that $\forall l_{t} \in T_{r_{i}} \quad \exists c_{\ell} \in C_{\ell_{i}}: \exists X:\left(\mathcal{R}\left(c_{\ell}, X\right) \wedge l_{t} \in X\right)$ is true in $\mathcal{S}(\phi)$. Let $C_{m_{i-1}}=\left\{c \ell^{\prime} \in C l \mid \exists l t^{\prime} \in T_{r_{i}} \cap B \square, O c\left(l t^{\prime}, c e^{\prime}\right)\right\}$ be the set of clauses such that the corresponding $\square$ clause is in $T r_{i}$. The world $w$ satisfies all clauses in $C_{\ell_{i}}$ and since $w$ is its own successor, it also satisfies all clauses in $C_{m_{i-1}}$. Hence, if $c_{\ell}$ is any clause in $C_{\ell_{i}} \cup C_{m_{i-1}}$, some literal occurring in $c_{\ell}$ must be satisfied in $w$. Therefore, $\forall c_{\ell} \in C_{\ell_{i}} \cup C_{m_{i-1}}:\left[\left(\exists l t \in T_{r_{i}}: O_{c}\left(c_{\ell}, l_{t}\right)\right) \vee\left(\exists l t \in L t \backslash T_{r_{i}}: \overline{O_{c}}\left(c_{\ell}, l_{t}\right)\right)\right]$ is true in $\mathcal{S}(\phi)$.

Let $l_{t}$ be any literal of the form $\diamond C N F$ in $T r_{i}$ and let $D m_{i-1}=\left\{c_{\ell} \in C_{l} \mid O_{c}\left(l_{t}, c_{\ell}\right)\right\}$ be the set of clauses in the corresponding $C N F$ formula. Since $w$ satisfies $l t$, there must be a successor $w^{\prime}$ of $w$ that satisfies all clauses in $D m_{i-1}$ and also all clauses in $C m_{i-1}$ since $w^{\prime}$ is a successor of $w$. Since all clauses in $D m_{i-1} \cup C m_{i-1}$ are at level at most $i-1$ and $w^{\prime}$ is a world in a reflexive Kripke model that satisfies all clauses in $D m_{i-1} \cup C m_{i-1}$, we can apply induction hypothesis to conclude that $\zeta[i-1]\left(D m_{i-1} \cup C m_{i-1}\right)$ is true in $\mathcal{S}(\phi)$.

Theorem 5.3. Given a modal CNF formula $\phi$, there is a FPT algorithm that checks if $\phi$ is satisfiable in reflexive models, with the treewidth of $\mathcal{S}(\phi)$ and the modal depth of $\phi$ as parameters.

Proof. Given $\phi, \mathcal{S}(\phi)$ can be constructed in polynomial time. To check that all clauses of $\phi$ at level $\operatorname{md}(\phi)$ are satisfiable in some world $w$ of some reflexive Kripke model $\mathcal{M}$, we check whether the formula $\exists C \ell_{\operatorname{md}(\phi)} \forall c_{\ell}\left(C_{\ell_{\operatorname{md}(\phi)}}\left(c_{\ell}\right) \Leftrightarrow\left(C l\left(c_{\ell}\right) \wedge L v_{\operatorname{md}(\phi)}\left(c_{\ell}\right)\right)\right) \wedge \zeta[\operatorname{md}(\phi)]\left(C_{\left.\ell_{\operatorname{md}(\phi)}\right)}\right)$ is true in $\mathcal{S}(\phi)$. By Lemma 5.2, this is possible iff $\phi$ is satisfiable in a reflexive model. The length of the above formula is linear in $\operatorname{md}(\phi)$. An application of Courcelle's theorem will give us the FPT algorithm.

\section{Transitive models}

In transitive models, formulae with small modal depth can check properties of all worlds reachable from a given world. To formalize this into a W[1]-hardness proof, we introduce the parameterized Partitioned Weighted Satisfiability (p-Pw-SAT ) problem. An instance of p-Pw-SAT problem is a triple $(\mathcal{F}$, part $: \Phi \rightarrow$ $[k], t g:[k] \rightarrow \mathbb{N}$ ), where $\mathcal{F}$ is a propositional CNF formula, part partitions the set of propositional variables into $k$ parts and we need to check if there is a satisfying assignment that sets exactly $\operatorname{tg}(p)$ variables to $T$ in each part $p$. Parameters are $k$ and pathwidth of the primal graph of $\mathcal{F}$ (one vertex for each propositional variable, an edge between two variables iff they occur together in a clause). The following lemma can be proved by a FPT reduction from the Number List Coloring Problem [11.

Lemma 6.1. The $p$-Pw-SAT problem is $\mathrm{W}[1]$-hard when parameterized by the number of parts $k$ and the pathwidth of the primal graph.

Proof. We will give a FPT reduction from the Number List Coloring Problem (NLCP). An instance of NLCP is a graph $G=(V, E)$, a set of colors $S_{v}$ for each vertex $v \in V$ and a target function $t g: \cup_{v \in V} S_{v} \rightarrow \mathbb{N}$. We need to check if $G$ can be properly colored (every adjacent pair of vertices get different colors) such that every vertex $v$ is colored from its set $S_{v}$ and there are exactly $t g(\ell)$ vertices colored with $\ell$ for every $\ell \in \cup_{v \in V} S_{v}$. In [11, it is proved that even for graphs of pathwidth 2, NLCP is W[1]-hard when parameterized by total number of colors in $\cup_{v \in V} S_{v}$. 
Given an instance of NLCP with a graph of pathwidth 2, we associate with it an instance of p-Pw-SAT with the set of propositional variables $\left\{q_{v}^{\ell} \mid v \in V, \ell \in S_{v}\right\}$. Every color $\ell \in \cup_{v \in V} S_{v}$ is a partition of the set of propositional variables and contains the variables $\left\{q_{v}^{\ell} \mid \ell \in S_{v}\right\}$. Target function is the same as target function of the NLCP instance. The CNF formula is the conjunction of the following formulae:

$$
\begin{aligned}
\text { atLeast } & \triangleq \bigwedge_{v \in V}\left(\bigvee_{\ell \in S_{v}} q_{v}^{\ell}\right) \\
\text { atMost } & \triangleq \bigwedge_{v \in V} \bigwedge_{\ell \neq \ell^{\prime} \in S_{v}}\left(\neg q_{v}^{\ell} \vee \neg q_{v}^{\ell^{\prime}}\right) \\
\text { proper } & \triangleq \bigwedge_{(v, u) \in E} \bigwedge_{\ell \in S_{v} \cap S_{u}}\left(\neg q_{v}^{\ell} \vee \neg q_{u}^{\ell}\right)
\end{aligned}
$$

Suppose the given NLCP instance is a Yes instance. In the associated p-PW-SAT instance, set $q_{v}^{\ell}$ to $T$ iff the vertex $v$ receives color $\ell$ in the witnessing coloring. Since every vertex gets a color from its set, the formula atLeast above is satisfied. Since every vertex gets at most one color, the formula atMost is satisfied. If $(v, u)$ is any edge in the graph, then since $v$ and $u$ get different colors in the witnessing coloring, the formula proper above is also satisfied. Since target function of the p-Pw-SAT instance is same as the target function of the NLCP instance, the target function of p-PW-SAT is also satisfied.

On the other hand, suppose that the instance of p-Pw-SAT is a YES instance. Color a vertex $v$ with the color $\ell$ iff the propositional variable $q_{v}^{\ell}$ is set to $T$ in the witnessing satisfying assignment. The formula atLeast ensures that every vertex gets at least one color from its set, while the formula at Most ensures that every vertex gets at most one color. If $(v, u)$ is an edge in $G$ and $\ell$ is a common color between $S_{v}$ and $S_{u}$, then the formula proper above ensures that at least one of the vertices $v, u$ do not get the color $\ell$. Hence, the coloring given to the graph $G$ is proper. Again since target function of the p-Pw-SAT instance is same as the target function of the NLCP instance, the target function of NLCP is also satisfied.

Now, it is left to prove that parameters of the p-Pw-SAT instance is bounded by some functions of the parameters of the NLCP instance. First parameter of the p-Pw-SAT instance is the number of partitions, which is same as the total number of colors in the NLCP instance (and later is a parameter of the NLCP instance). Second parameter is the pathwidth of the primal graph of the CNF formula. Consider any path decomposition of width 2 of the graph $G$ in the NLCP instance. For every bag $B$ and every vertex $v$ in the bag, replace $v$ by the set $\left\{q_{v}^{\ell} \mid \ell \in S_{v}\right\}$. We claim that the resulting decomposition is a path decomposition of the primal graph of the CNF formula in the p-Pw-SAT instance. It is sufficient to prove that for every clause in the CNF formula, there is a bag containing all propositional variables occurring as literals in that clause. For any clause in the formula atLeast or atMost associated with a vertex $v$, any bag that contained the vertex $v$ before replacement will meet the above criteria. For a clause in the formula proper associated with an edge $(v, u)$, any bag that contained the vertices $v$ and $u$ before replacement will suffice. In the new path decomposition, number of elements in any bag is at most 3 times the total number of colors in the NLCP instance. Hence, the pathwidth of the primal graph of the CNF formula in the p-Pw-SAT instance is also bounded by a function of the parameters of the NLCP instance.

Theorem 6.2. With treewidth and modal depth as parameters, modal satisfiability in transitive models is $\mathrm{W}[1]$-hard.

The rest of this section is devoted to a proof of the above theorem, which is by a FPT reduction from p-PwSAT to satisfiability of modal CNF formulae in transitive models. Given an instance $(\mathcal{F}$, part $: \Phi \rightarrow[k], t g:$ $[k] \rightarrow \mathbb{N}$ ) of p-Pw-SAT problem with the pathwidth of the primal graph of $\mathcal{F}$ being $p w$, we construct a modal CNF formula $\phi_{\mathcal{F}}$ of modal depth 2 in FPT time such that the pathwidth (and hence the treewidth) of $\mathcal{S}\left(\phi_{\mathcal{F}}\right)$ is bounded by a function of $p_{w}$ and $k$ and p-PW-SAT is a YES instance iff $\phi_{\mathcal{F}}$ is satisfiable in a transitive model. Suppose the propositional variables used in $\mathcal{F}$ are $q_{1}, q_{2}, \ldots, q_{n}$. The idea is that if $\phi_{\mathcal{F}}$ is satisfied at some world $w_{0}$ in some transitive model $\mathcal{M}$, then $\mathcal{M}, w_{0} \models \mathcal{F}$. To check that the required targets of the number of variables set to true in each partition are met, $\phi_{\mathcal{F}}$ will force the existence of worlds $w_{1}, w_{2}, \ldots, w_{n}$ arranged as $w_{0} \mapsto w_{1} \mapsto w_{2} \mapsto \cdots \mapsto w_{n}$. In the formula $\phi_{\mathcal{F}}$, we will maintain a counter for each partition of the propositional variables. At each world $w_{i}$, if $q_{i}$ is true, we will force the counter corresponding to $\operatorname{part}\left(q_{i}\right)$ to increment. At the world $w_{n}$, the counters will have the number of variables set to $T$ in each partition. We will then verify in the formula $\phi_{\mathcal{F}}$ that these counts meet the given target. Such counting tricks have come under standard usage in complexity theoretic arguments of modal logic. The challenge here is to implement the counting in a modal formula of small pathwidth. 
In a p-Pw-SAT instance containing $n$ propositional variables and $k$ partitions, we will denote the number of variables in partition $p$ by $n[p]$. We first construct an optimal path decomposition of the primal graph of $\mathcal{F}$ in FPT time. We will name the variables occurring in the first bag as $q_{1}, \ldots, q_{i}$. We will name the variables newly introduced in the second bag as $q_{i+1}, \ldots, q_{i^{\prime}}$ and so on. In the rest of the construction, we will use this same ordering $q_{1}, \ldots, q_{n}$ of the propositional variables. This will be important to maintain the pathwidth of the resulting modal formula low. The modal CNF formula $\phi_{\mathcal{F}}$ will use all the propositional variables $q_{1}, \ldots, q_{n}$ used by $\mathcal{F}$ and also use the following additional variables:

$-t \uparrow_{1}, \ldots, t \uparrow_{k}, f \uparrow_{1}, \ldots, f \uparrow_{k}$ : partition indicators.

- For each partition $p, \operatorname{tr}_{p}^{0}, \ldots, \operatorname{tr}_{p}^{n[p]}, f l_{p}^{0}, \ldots, f l_{p}^{n[p]}$ : counters to count the number of variables set to $\top$ and $\perp$ in partition $p$.

$-d_{0}, \ldots, d_{n+1}:$ depth indicators.

The modal CNF formula $\phi_{\mathcal{F}}$ is the conjunction of the formulae described below. For clarity, we have used the shorthand notation $\Rightarrow$ but they can be easily converted to CNF. Also for notational convenience, we will use $\operatorname{part}(i)$ instead of $\operatorname{part}\left(q_{i}\right) . \Phi(p)$ is the set of variables among $\left\{q_{1}, \ldots, q_{n}\right\}$ in partition $p$. The formula determined ensures that all successors of $w_{0}$ preserve the assignment of $q_{1}, \ldots, q_{n}$. The formula depth ensures that for all $i, d_{i} \wedge \neg d_{i+1}$ holds in the world $w_{i}$.

In $w_{i-1}$, if $q_{i}$ is set to $T$, we want to indicate that in $w_{i}$, the counter for partition $\operatorname{part}(i)$ should be incremented. We will indicate this in the formula setCounter by setting the variable $t_{\uparrow}$ part(i) to $T$. Similar indication is done for the counter keeping track of variables set to $\perp$ in partition $p$.

$$
\begin{aligned}
& \text { determined } \triangleq \bigwedge_{i=1}^{n} q_{i} \Rightarrow \square q_{i} \wedge \bigwedge_{i=1}^{n} \neg q_{i} \Rightarrow \square \neg q_{i} \\
& \text { depth } \triangleq \diamond\left(d_{1} \wedge \neg d_{2}\right) \wedge \bigwedge_{i=1}^{n-1} \square\left[\left(d_{i} \wedge \neg d_{i+1}\right) \Rightarrow \diamond\left(d_{i+1} \wedge \neg d_{i+2}\right)\right] \\
& \text { setCounter } \triangleq\left(q_{1} \Rightarrow t \uparrow \operatorname{part(1)}\right) \wedge\left(\neg q_{1} \Rightarrow f \uparrow \operatorname{part}(1)\right) \\
& \wedge \bigwedge_{i=2}^{n} \square\left\{\left[d_{i-1} \wedge \neg d_{i}\right] \Rightarrow\left[\left(q_{i} \Rightarrow t \uparrow_{\text {part }(i)}\right) \wedge\left(\neg q_{i} \Rightarrow f \uparrow_{\text {part }(i)}\right)\right]\right\} \\
& \text { incCounter } \triangleq\left(t_{\uparrow_{\text {part }(1)}} \Rightarrow \square t r_{\text {part (1) }}^{1}\right) \wedge\left(f \uparrow_{\text {part }(1)} \Rightarrow \square f l_{\text {part (1) }}^{1}\right) \\
& \wedge \bigwedge_{p=1}^{k} \bigwedge_{j=0}^{n[p]-1} \square\left[t \uparrow_{p} \Rightarrow\left(t_{p}^{j} \Rightarrow \square t r_{p}^{j+1}\right)\right] \wedge \square\left[f \uparrow_{p} \Rightarrow\left(f l_{p}^{j} \Rightarrow \square f l_{p}^{j+1}\right)\right] \\
& \text { targetMet } \triangleq \bigwedge_{p=1}^{k} \square\left[d_{n} \Rightarrow\left(\operatorname{tr}_{p}^{t g(p)} \wedge \neg t_{p}^{t g(p)+1}\right)\right] \\
& \wedge \bigwedge_{p=1}^{k} \square\left[d_{n} \Rightarrow\left(f l_{p}^{n[p]-t g(p)} \wedge \neg f l_{p}^{n[p]-t g(p)+1}\right)\right]
\end{aligned}
$$

Variables $t_{p}^{0}, \ldots, t_{r}^{n[p]}$ implement the counter keeping track of variables set to $\top$ in partition $p$. If $j$ variables in $\Phi(p) \cap\left\{q_{1}, \ldots, q_{i}\right\}$ are set to $\top$, then we want $t_{p}^{j}$ to be set to $\top$ in $w_{i}$. To maintain this, in $w_{i-1}$, if it is indicated that a counter is to be incremented (by setting $t_{\uparrow}$ to $T$ ), we will force all successors of $w_{i-1}$ to increment the $t_{r}$ counter in the formula incCounter. Finally, we check that at $w_{n}$, all the targets are met in the formula targetMet.

The modal CNF formula $\phi_{\mathcal{F}}$ we need is the conjunction of $\mathcal{F}$, the formulae defined above and the miscellaneous formulae below (which ensure that counters are initiated properly and are monotonically non- 
decreasing).

$$
\begin{aligned}
\text { determined } & \triangleq \bigwedge_{p=1}^{k} t r_{p}^{0} \Rightarrow \square t r_{p}^{0} \wedge \bigwedge_{p=1}^{k} f l_{p}^{0} \Rightarrow \square f l_{p}^{0} \\
\text { countInit } & \triangleq d_{0} \wedge \neg d_{1} \wedge \bigwedge_{p=1}^{k}\left(\neg t r_{p}^{1} \wedge \neg f l_{p}^{1} \wedge t r_{p}^{0} \wedge f l_{p}^{0}\right) \\
\text { depth } & \triangleq \bigwedge_{p=1}^{k} \bigwedge_{j=0}^{n[p]}\left[\square\left(t r_{p}^{j} \Rightarrow \square t r_{p}^{j}\right) \wedge \square\left(f l_{p}^{j} \Rightarrow \square f l_{p}^{j}\right)\right] \\
\text { countMonotone } & \triangleq \bigwedge_{i=1}^{n} \square\left(d_{i} \Rightarrow d_{i-1}\right) \wedge \bigwedge_{p=1}^{k} \bigwedge_{j=2}^{n[p]}\left[\square\left(t r_{p}^{j} \Rightarrow t r_{p}^{j-1}\right) \wedge \square\left(f l_{p}^{j} \Rightarrow f l_{p}^{j-1}\right)\right]
\end{aligned}
$$

Lemma 6.3. If a p-PW-SAT instance is a YES instance, then the modal formula constructed above is satisfied in a transitive Kripke model.

Proof. We will construct a transitive Kripke model using the satisfying assignment $f$ that satisfies $\mathcal{F}$ while meeting the given target. The model $\mathcal{M}$ consists of worlds $w_{0}, w_{1}, \ldots, w_{n}$ arranged as $w_{0} \mapsto w_{1} \mapsto w_{2} \mapsto$ $\cdots \mapsto w_{n}$. In all worlds, $q_{i}$ is set to $f\left(q_{i}\right)$ for all $i$, thus ensuring that $\mathcal{M}, w_{0} \models \mathcal{F} \wedge$ determined. In $w_{i},\left\{d_{0}, \ldots, d_{i}\right\}$ are set to $\top$ and $\left\{d_{i+1}, \ldots, d_{n+1}\right\}$ are set to $\perp$ for all $i$ between 0 and $n$, thus ensuring that $\mathcal{M}, w_{0} \models$ depth $\wedge d_{0} \wedge \neg d_{1}$, the last two clauses coming from the formula depth'. It also ensures that $\mathcal{M}, w_{0} \models \bigwedge_{i=1}^{n} \square\left(d_{i} \Rightarrow d_{i-1}\right)$, which is part of countMonotone. We will set $t r_{p}^{0}$ and $f l_{p}^{0}$ to $\top$ in all worlds and $t_{p}^{1}$ and $f l_{p}^{1}$ to $\perp$ in $w_{0}$ for all partitions $p$, thus ensuring $\mathcal{M}, w_{0} \models$ countInit $\wedge$ determined ${ }^{\prime}$. At $w_{i-1}$, we will set $t \uparrow_{\text {part }(i)}$ to $q_{i}$ 's value in the same world and $f \uparrow_{\text {part }(i)}$ to $\neg q_{i}$ 's value. This will ensure that $\mathcal{M}, w_{0} \models$ setCounter.

At $w_{i}$, for any partition $p$, if $j$ variables in $\Phi(p) \cap\left\{q_{1}, \ldots, q_{i}\right\}$ are set to $\top$, then we will set $\left\{\operatorname{tr}_{p}^{0}, \ldots, t r_{p}^{j}\right\}$ to $\top$ and $\left\{t_{p}^{j+1}, \ldots, t r_{p}^{n[p]}\right\}$ to $\perp$. If $j^{\prime}$ variables in $\Phi(p) \cap\left\{q_{1}, \ldots, q_{i}\right\}$ are set to $\perp$, we will set $\left\{f l_{p}^{0}, \ldots, f l_{p}^{j^{\prime}}\right\}$ to true and $\left\{f l_{p}^{j^{\prime}+1}, \ldots, f_{l_{p}^{n}}^{n[p]}\right\}$ to $\perp$. For any $p \neq \operatorname{part}(i+1)$, we will set $t \uparrow_{p}$ and $f_{\uparrow_{p}}$ to $\perp$ at $w_{i}$. These will ensure that $\mathcal{M}, w_{0} \models$ incCounter $\wedge$ depth' $\wedge$ countMonotone.

Combined with the above settings of all propositional variables in $\mathcal{M}$, it is easy to check that the fact that $f$ meets the target for each partition implies $\mathcal{M}, w_{0} \models$ target Met.

Lemma 6.4. Suppose the modal CNF formula $\phi_{\mathcal{F}}$ constructed above is satisfied at some world $w_{0}$ of some transitive Kripke model $\mathcal{M}$. Then $\mathcal{M}$ contains distinct worlds $w_{1}, \ldots, w_{n}$ such that for each $i$ between 1 and $n, w_{i}$ is a successor of $w_{i-1}$. Moreover, $\left\{d_{0}, \ldots, d_{i}\right\}$ are set to $\top$ and $\left\{d_{i+1}, \ldots, d_{n+1}\right\}$ are set to $\perp$ in $w_{i}$. For any partition $p$, if $j$ variables in $\Phi(p) \cap\left\{q_{1}, \ldots, q_{i}\right\}$ are set to $\top$ in $w_{0}$, then $\left\{t_{p}^{0}, \ldots, t_{p}^{j}\right\}$ are all set to $\top$ in $w_{i}$. If $j^{\prime}$ variables in $\Phi(p) \cap\left\{q_{1}, \ldots, q_{i}\right\}$ are set to $\perp$ in $w_{0}$, then $\left\{f l_{p}^{0}, \ldots, f l_{p}^{j^{\prime}}\right\}$ are all set to $\top$ in $w_{i}$.

Proof. We will first prove the existence of worlds $w_{1}, \ldots, w_{i}$ by induction on $i$.

Base case $i=1$ : Since $\mathcal{M}, w_{0} \models d e p t h$, there must be a successor $w_{1}$ of $w_{0}$ that satisfies $d_{1} \wedge \neg d_{2}$. Since $\mathcal{M}, w_{0} \models$ countInit, $w_{0}$ satisfies $d_{0} \wedge \neg d_{1}$ and hence $w_{1}$ can not be same as $w_{0}$. Since $\mathcal{M}, w_{0} \models \square\left(d_{3} \Rightarrow d_{2}\right)$ (part of countMonotone) and $w_{1}$ is a successor of $w_{0}$, we get $\mathcal{M}, w_{1} \models d_{3} \Rightarrow d_{2}$. Since $d_{2}$ is set to $\perp$ in $w_{1}$, this means that $d_{3}$ is also set to $\perp$ in $w_{1}$. Similar reasoning can be used to prove that all of $\left\{d_{2}, \ldots, d_{n+1}\right\}$ are set to $\perp$ in $w_{1}$. The fact that $\mathcal{M}, w_{1} \models d_{1} \Rightarrow d_{0}$ means that $d_{0}$ is set to $\top$ in $w_{1}$ (since $d_{1}$ is set to $\top$ in $\left.w_{1}\right)$.

Induction step: Assume that worlds $w_{1}, \ldots, w_{i}$ exist in $\mathcal{M}$ with the stated properties. Hence, $w_{i}$ satisfies $d_{i} \wedge \neg d_{i+1}$. Since $w_{0}$ satisfies depth and $w_{i}$ is a successor of $w_{0}$ (by transitivity), there must be a successor $w_{i+1}$ of $w_{i}$ that satisfies $d_{i+1} \wedge \neg d_{i+2}$. Since all worlds $w_{0}, \ldots, w_{i}$ satisfy $\neg d_{i+1}, w_{i+1}$ is distinct from all of them. The fact that $w_{i+1}$ satisfies $d_{i^{\prime}} \Rightarrow d_{i^{\prime}-1}$ for all $i^{\prime}$ (these formulae are part of countMonotone formula satisfied by $w_{0}$ ) can be used to show that all of $d_{0}, \ldots, d_{i+1}$ are set to $T$ in $w_{i+1}$ and all of $d_{i+2}, \ldots, d_{n+1}$ are set to $\perp$ in $w_{i+1}$.

We will now prove the second claim of the lemma, which is about values of $\left\{\operatorname{tr}_{p}^{0}, \ldots, t_{p}^{j}\right\}$ in $w_{i}$. We will first prove that $t r_{p}^{j}$ is set to $T$ by induction on $i$.

Base case $i=1$ : If $q_{1}$ is not in part $p$, there is nothing to prove ( $\operatorname{tr}_{\text {part }(1)}^{0}$ is set to $\top$ in all worlds). If $q_{1}$ is in part $p$ and $q_{1}$ is set to $\perp$, there is nothing to prove. If $q_{1}$ is in part $p$ and $q_{1}$ is set to $\top$, then since $w_{0}$ 
satisfies setCounter, we get $\mathcal{M}, w_{0} \models q_{1} \Rightarrow t \uparrow_{p a r t(1)}$. Since, $q_{1}$ is set to $\top$ and $\operatorname{part}(1)=p$, we get that $t \uparrow_{p}$ is set to $\top$ in $w_{0}$. Since $w_{0}$ satisfies incCounter, we get $\mathcal{M}, w_{0} \models t \uparrow_{p} \Rightarrow \square t r_{p}^{1}$ and hence $\mathcal{M}, w_{0} \models \square t r_{p}^{1}$. Since $w_{1}$ is a successor of $w_{0}$, we conclude that in $w_{1}, t_{r}^{1}$ is set to $\top$.

Induction step: Case 1: $q_{i}$ is not in part $p$ and none of the variables in $\Phi(p) \cap\left\{q_{1}, \ldots, q_{i}\right\}$ are set to to $\top$ in $w_{i}$. In this case, there is nothing to prove.

Case 2: $q_{i}$ is not in part $p$ and some $1 \leq j<i$ variables in $\Phi(p) \cap\left\{q_{1}, \ldots, q_{i}\right\}$ are set to $T$. By the induction hypothesis, $t r_{p}^{j}$ is set to $\top$ in $w_{i-1}$. Now $\mathcal{M}, w_{0} \models \operatorname{depth}$. Hence $\mathcal{M}, w_{0} \models \square\left(t r_{p}^{j} \Rightarrow \square t t_{p}^{j}\right)$, and hence $\mathcal{M}, w_{i-1} \models t r_{p}^{j} \Rightarrow \square t r_{p}^{j}$ (since $w_{i-1}$ is a successor of $w_{0}$ ), and hence $\mathcal{M}, w_{i-1} \models \square t r_{p}^{j}$ (since $t_{p}^{j}$ is set to $\top$ in $w_{i-1}$ ), and hence $\mathcal{M}, w_{i} \models t_{p}^{j}$ (since $w_{i}$ is a successor of $w_{i-1}$ ).

Case 3: $q_{i}$ is in part $p$ and $q_{i}$ is set to $\perp$. If none of the variables in $\Phi(p) \cap\left\{q_{1}, \ldots, q_{i}\right\}$ are set to $T$, then the argument is similar to case 1 . If some $1 \leq j<i$ variables in $\Phi(p) \cap\left\{q_{1}, \ldots, q_{i}\right\}$ are set to $T$, then the argument is similar to case 2 .

Case 4: $q_{i}$ is in part $p$ and $q_{i}$ is set to $T$. We know that $w_{i-1}$ satisfies $d_{i-1} \wedge \neg d_{i}$. Since $w_{0}$ satisfies setCounter, we have $\mathcal{M}, w_{0} \models \square\left\{\left[d_{i-1} \wedge \neg d_{i}\right] \Rightarrow\left[q_{i} \Rightarrow t t_{\text {part }(i)}\right]\right\}$, and hence $\mathcal{M}, w_{i-1} \models\left[d_{i-1} \wedge \neg d_{i}\right] \Rightarrow$ $\left[q_{i} \Rightarrow t \uparrow_{p a r t(i)}\right]$ (since $w_{i-1}$ is a successor of $w_{0}$ ), and hence $\mathcal{M}, w_{i-1} \models q_{i} \Rightarrow t \uparrow_{p}$ (since $\mathcal{M}, w_{i-1} \models d_{i-1} \wedge \neg d_{i}$ ), and hence $\mathcal{M}, w_{i-1} \models t \uparrow_{p}$ ( since $\mathcal{M}, w_{i-1} \models q_{i}$ ). Since $w_{0}$ satisfies incCounter and $w_{i-1}$ is a successor of $w_{0}$, we get $\mathcal{M}, w_{i-1} \models t \uparrow_{p} \Rightarrow\left(t r_{p}^{j-1} \Rightarrow \square t r_{p}^{j}\right)$. We have already seen that $t \uparrow_{p}$ is set to $\top$ in $w_{i-1}$ and $t r_{p}^{j-1}$ is set to $\top$ in $w_{i-1}$ by the induction hypothesis ( $j$ is at least 1 since $q_{i}$ is in part $p$ and is set to $T$ ). Hence, we get $\mathcal{M}, w_{i-1} \models \square t_{p}^{j}$. Since $w_{i}$ is a successor of $w_{i-1}$, we conclude that $t_{p}^{j}{ }_{p}^{j}$ is set to $\top$ in $w_{i}$.

Now, since $w_{0}$ satisfies $\square\left(t r_{p}^{j} \Rightarrow t r_{p}^{j-1}\right)$ (this is part of countMonotone) and $w_{i}$ is a successor of $w_{0}$, we get $\mathcal{M}, w_{i} \models t r_{p}^{j} \Rightarrow t r_{p}^{j-1}$. Since $t_{p}^{j}$ is set to $\top$ in $w_{i}$, it follows that $t r_{p}^{j-1}$ is also set to $\top$ in $w_{i}$. Similarly, $t_{p}^{0}, \ldots, t r_{p}^{j}$ are all set to $\top$ in $w_{i}$.

The proof for values of $\left\{f l_{p}^{0}, \ldots, f l_{p}^{j^{\prime}}\right\}$ is symmetric to the proof of values of $\left\{\operatorname{tr}_{p}^{0}, \ldots, t r_{p}^{j}\right\}$.

Theorem 6.5. If $\phi_{\mathcal{F}}$ constructed above is satisfied in a transitive model, then the p-PW-SAT instance is a YES instance.

Proof. Suppose $\phi_{\mathcal{F}}$ is satisfied in some world $w_{0}$ of a transitive model. Since $\mathcal{F}$ is part of $\phi_{\mathcal{F}}$, the assignment to $\left\{q_{1}, \ldots, q_{n}\right\}$ induced by $w_{0}$ satisfies $\mathcal{F}$. We claim that this assignment also meets the targets. If not, we will derive a contradiction. For some partition $p$, suppose there are more than $\operatorname{tg}(p)$ variables set to $\top$. Then by Lemma 6.4. $\operatorname{tr}_{p}^{t g(p)+1}$ will be set to $\top$ in $w_{n}$, contradicting the fact that $w_{0}$ satisfies target Met. For some partition $p$, if there are less than $\operatorname{tg}(p)$ variables set to $\top$, then there will be more than $n[p]-\operatorname{tg}(p)$ variables set to $\perp$. By Lemma $6.4 \operatorname{tr}_{p}^{n[p]-\operatorname{tg}(p)+1}$ will be set to $\top$ in $w_{n}$, again contradicting the fact that $w_{0}$ satisfies targetMet.

Given an instance of p-PW-SAT problem, the formula $\phi_{\mathcal{F}}$ described above can be constructed in FPT time. To complete the proof of Theorem 6.2, we will prove that the pathwidth of $\phi_{\mathcal{F}}$ is bounded by some function of $k$ and $p_{w} . \phi_{\mathcal{F}}$ has been carefully constructed to keep pathwidth low.

Lemma 6.6. Pathwidth of $\mathcal{S}\left(\phi_{\mathcal{F}}\right)$ is at most $4 p w+2 k+5$.

Proof. Given an optimal path decomposition of the primal graph of $\mathcal{F}$, depth counters can be added to the bags without increasing their size much since the order of depth counters is same as the order of $q_{1}, \ldots, q_{n}$. There are only $2 k$ partition indicators $t_{\uparrow_{1}}, \ldots, t \uparrow_{k}, f \uparrow_{1}, \ldots, f \uparrow_{k}$, so they can also be added to the bags without increasing their size very much. However, the set of $2 n$ partition counters (of the form $t r_{p}^{j}$ or $f l_{p}^{j}$ ) has to be added carefully to maintain the size of the bags. Formulas of $\phi_{\mathcal{F}}$ have been carefully designed to enable this. The key observation is that the only "link" between $q_{1}, \ldots, q_{n}$ and partition counters are partition indicators and there are only $2 k$ of them. The following proof relies on this observation.

Consider an optimal path decomposition of the primal graph of $\mathcal{F}$ with each bag containing at most $p w$ elements. Ensure that for all $i$ with $1 \leq i<n$, there is a bag containing both $q_{i}$ and $q_{i+1}$ or there is a bag with $q_{i}$ such that the next bag contains $q_{i+1}$ (call this the continuity property). If this is not the case for some $i$, consider the last bag $B$ containing $q_{i}$ and the first bag $B^{\prime}$ containing $q_{i+1}$. No bag that is between $B$ and $B^{\prime}$ will introduce any new variable (if it did, that new variable would have been $q_{i+1}$ according to our order). Hence, all the bags in between $B$ and $B^{\prime}$ are subsets of $B$. Hence, they can all be removed and $B^{\prime}$ can become the bag immediately after $B$. The resulting decomposition is still a path decomposition of the primal graph of $\mathcal{F}$ with each bag containing at most $p w$ elements. Moreover, the order of variables $q_{1}, \ldots, q_{n}$ does not change due to the change we have made in the path decomposition. This new decomposition has a 
bag containing $q_{i}$ such that the next bag contains $q_{i+1}$. Now, we can repeat the above process until we get a path decomposition with the continuity property.

For any $i$ with $1 \leq i \leq n$, let $B_{i}$ be a bag containing the propositional variable $q_{i}$. We will expand this path decomposition by adding variables used in $\phi_{\mathcal{F}}$ such that for every clause that appears in $\phi_{\mathcal{F}}$, there is a bag that contains all propositional variables appearing in that clause. Each of these expanded bags will have at most $4 p w+2 k$ elements. We will then show how to expand this into a path decomposition of $\mathcal{S}\left(\phi_{\mathcal{F}}\right)$, by adding at most 6 elements to each bag (creating duplicate copies of existing bags if required). This will prove that the pathwidth of $\mathcal{S}\left(\phi_{\mathcal{F}}\right)$ is at most $4 p w+2 k+5$.

First, in each bag $B$ and each element $q_{i}$ in it, add $d_{i-1}, d_{i}$ and $d_{i+1}$. Note that due to continuity property of the decomposition we started with, the expanded decomposition still retains the property that all bags containing an element forms a connected component, even after adding depth counters $d_{0}, \ldots, d_{n+1}$. Next, add $t \uparrow_{1}, \ldots, t \uparrow_{k}, f \uparrow_{1}, \ldots, f \uparrow_{k}$ to all the bags. We will refer to the bag containing $q_{i}, d_{i-1}, d_{i}$ and $d_{i+1}$ as $B_{i}$. Now, we have a decomposition with each bag containing at most $4 p w+2 k$ elements, and the last bag contains $d_{n}$. To this bag, we will append $2 k$ paths serially. For $1 \leq p \leq k,(2 p-1)^{\text {th }}$ path will be as follows: $\left\{d_{n}, t \uparrow_{1}, \ldots, t \uparrow_{k}, f \uparrow_{1}, \ldots, f \uparrow_{k}, t r_{p}^{0}, t r_{p}^{1}\right\}-\left\{d_{n}, t \uparrow_{1}, \ldots, t \uparrow_{k}, f \uparrow_{1}, \ldots, f \uparrow_{k}, t r_{p}^{1}, t r_{p}^{2}\right\}-\cdots-$ $\left\{d_{n}, t \uparrow_{1}, \ldots, t \uparrow_{k}, f \uparrow_{1}, \ldots, f \uparrow_{k}, t_{p}^{n[p]-1}, t_{p}^{n[p]}\right\}$. We will refer to these bags as $B_{p}^{1}, \ldots, B_{p}^{n[p]} .2 p^{\text {th }}$ path is similar, with $f l_{p}$ variables replacing $t_{r}$ variables. We will refer to these bags in $2 p^{\text {th }}$ path as $B_{p}^{1^{\prime}}, \ldots, B_{p}^{n[p]^{\prime}}$. Each of these new bags has at most $2 k+3$ elements, and the whole decomposition still retains the property that for any element, the set of bags containing that element forms a connected component.

Now we will show how to expand the above decomposition into a path decomposition of $\mathcal{S}\left(\phi_{\mathcal{F}}\right)$. We have to add clauses and literals occurring in $\phi_{\mathcal{F}}$ and ensure that for any pair of elements $O_{c}\left(e_{1}, e_{2}\right)$ or $\overline{O_{c}}\left(e_{1}, e_{2}\right)$, there is a bag containing both $e_{1}$ and $e_{2}$. To achieve this, we may have to "augment" an existing bag with new elements. If $B_{i}$ is a bag in the path decomposition $\cdots-B_{i}-\ldots$, augmenting $B_{i}$ with elements $e_{1}$ and $e_{2}$ means that we add another bag $\cdots-B_{i}^{\prime}-B_{i}-\ldots$ with $B_{i}^{\prime}$ containing all elements of $B_{i}$ and in addition containing $e_{1}$ and $e_{2}$. If we ensure that these new elements introduced during augmentation is never added to any other bag in the decomposition, augmentation will not violate the path decomposition's property that for any element, the set of bags containing that element forms a connected component. Now, we will go through each sub-formula of $\phi_{\mathcal{F}}$ and prove that all its clauses, literals and $O_{c}$ pairs are already represented in the path decomposition we have constructed above or that the decomposition can be augmented to represent them.

- Clauses in $\mathcal{F}$ : For each clause in $\mathcal{F}$, the propositional variables in that clause form a clique in the primal graph of $\mathcal{F}$. Hence, there is a bag $B$ in the new decomposition that contains all propositional variables occurring in that clause. Augment $B$ with a new domain element representing the clause.

- determined: Here, the clauses are of the form $\neg q_{i} \vee \square q_{i}$ and $q_{i} \vee \square \neg q_{i}$. Augment the bag $B_{i}$ containing $q_{i}$ with 3 domain elements, one for the clause $\neg q_{i} \vee \square q_{i}$ itself, one for the literal $\square q_{i}$ and one for the clause in this literal that contains $q_{i}$ as its only literal. Perform similar augmentation for the clause $q_{i} \vee \square \neg q_{i}$.

- depth: For $\diamond\left(d_{1} \wedge \neg d_{2}\right)$, augment the bag $B_{1}$ containing $d_{1}$ and $d_{2}$ with 4 domain elements representing literals and clauses of $\diamond\left(d_{1} \wedge \neg d_{2}\right)$. Augment the bag $B_{i+1}$ containing $d_{i}, d_{i+1}$ and $d_{i+2}$ with 6 elements representing literals and clauses of $\square\left[\neg d_{i} \vee d_{i+1} \vee \diamond\left(d_{i+1} \wedge \neg d_{i+2}\right)\right]$.

- setCounter: Augment the bag $B_{1}$ containing $q_{1}$ and $t \uparrow_{\text {part }(1)}$ with one element representing the clause $\neg q_{1} \vee t \uparrow_{\text {part }(1)}$. Do a similar augmentation for the clause $q_{1} \vee f \uparrow_{\text {part }(1)} . \square(q \wedge r)$ is equivalent to $\square q \wedge$ $\square r$. Hence, the latter part of setCounter can be split into clauses $\square\left(\neg d_{i-1} \vee d_{i} \vee \neg q_{i} \vee t t_{\text {part }(i)}\right)$ and $\square\left(\neg d_{i-1} \vee d_{i} \vee q_{i} \vee f \uparrow_{\text {part }(i)}\right)$. Augment the bag $B_{i}$ containing $d_{i-1}, d_{i}, q_{i}, t_{\text {part }(i)}$ and $f_{\uparrow \text { part }(i)}$ with 6 elements representing clauses and literals of these two clauses.

- incCounter: Augment the bag $B_{\text {part(1) }}^{1}$ containing $t \uparrow_{\text {part(1) }}$ and $\operatorname{tr}_{\text {part(1) }}^{1}$ with 3 elements representing clauses and literals of $\left(\neg t \uparrow_{\text {part }(1)} \vee \square t r_{\text {part (1) }}^{1}\right)$. Similarly augment the bag $B_{\text {part }(1)}^{1^{\prime}}$ for $\left(\neg f \uparrow_{\text {part }(1)} \vee\right.$ $\left.\square f l_{\text {part (1) }}^{1}\right)$. Augment the bag $B_{p}^{j+1}$ containing $t \uparrow_{p}, t_{p}^{j}$ and $t_{p}^{j+1}$ with 6 elements representing literals and clauses of $\square\left(\neg t \uparrow_{p} \vee \neg t r_{p}^{j} \vee \square t r_{p}^{j+1}\right)$. Similarly augment $B_{p}^{j+1^{\prime}}$ for $\square\left(\neg f \uparrow_{p} \vee \neg f l_{p}^{j} \vee \square f l_{p}^{j+1}\right)$.

- targetMet: Augment the bag $B_{p}^{t g(p)+1}$ containing $d_{n}, \operatorname{tr}_{p}^{t g(p)}$ and $\operatorname{tr}_{p}^{t g(p)+1}$ with 6 elements for the literals and clauses in $\square\left(\neg d_{n} \vee t_{p}^{t g(p)}\right)$ and $\square\left(\neg d_{n} \vee \neg t_{p}^{t g}{ }^{t p)+1}\right)$. Similarly augment $B_{p}^{n[p]-t g(p)+1^{\prime}}$ for $\square\left(\neg d_{n} \vee\right.$ $\left.f_{l}^{n[p]-t g(p)}\right)$ and $\square\left(\neg d_{n} \vee \neg f l_{p}^{n[p]-t g(p)+1}\right)$

- determined': Augment the bag $B_{p}^{1}$ containing $\operatorname{tr}_{p}^{0}$ with 3 elements representing literals and clauses of $\neg t r_{p}^{0} \vee \square t r_{p}^{0}$. Similarly augment $B_{p}^{1^{\prime}}$ for $\neg f l_{p}^{0} \vee \square f l_{p}^{0}$. 
- countInit: Augment the bag $B_{1}$ containing $d_{0}$ and $d_{1}$ with 2 elements representing the clauses in $d_{0} \wedge \neg d_{1}$. Augment the bag $B_{p}^{1}$ containing $t_{p}^{0}$ and $t r_{p}^{1}$ with 2 elements representing the clauses in $\neg t_{p}^{1} \wedge t r_{p}^{0}$. Similarly augment $B_{p}^{1^{\prime}}$ for $\neg f l_{p}^{1} \wedge f l_{p}^{0}$.

- depth': Augment the bag $B_{p}^{j}$ containing $t_{r}^{j}$ with 6 elements representing literals and clauses of $\square\left(\neg t r_{p}^{j} \vee\right.$ $\left.\square t r_{p}^{j}\right)$. Similarly augment $B_{p}^{j^{\prime}}$ for $\square\left(\neg f l_{p}^{j} \vee \square f l_{p}^{j}\right)$.

- countMonotone: Augment the bag $B_{i}$ containing $d_{i}$ and $d_{i-1}$ with 3 elements representing literals and clauses of $\square\left(\neg d_{i} \vee d_{i-1}\right)$. Augment the bag $B_{p}^{j}$ containing $t_{p}^{j}$ and $t r_{p}^{j-1}$ with 3 elements representing literals and clauses of $\square\left(\neg t r_{p}^{j} \vee t r_{p}^{j-1}\right)$. Similarly augment $B_{p}^{j^{\prime}}$ for $\square\left(\neg f_{p}^{j} \vee f_{l}^{j-1}\right)$.

In the absence of transitivity, the above reduction would require a formula of modal depth that depends on $n$ (and hence it would no longer be a FPT reduction). The above hardness proof will however go through for any class of transitive frames that has paths of unbounded length of the form $w_{1} \mapsto w_{2} \mapsto \cdots \mapsto w_{n}$ without any reverse path 2 . See [31] for some context on such classes of transitive frames of unbounded depth. Readers familiar with frames with no branching to the right (axiom .3) may infer that the above hardness proof will also go through for K4.3 frames.

\section{Conclusions and Future Work}

By expressing satisfiability of modal formulae as a MSO property, we obtained a FPT algorithm for modal satisfiability in general models with treewidth and modal depth as parameters. Due to the dependence of the constructed MSO sentence on modal depth, the FPT algorithm obtained in section 3 has a running time with a tower of 2's whose height is $\mathcal{O}(\operatorname{md}(\phi))$. Unless, PTIME $=\mathrm{NP}$, such dependence on modal depth cannot be avoided due to the following observation. In [1, Lemma 1], it is shown how to encode an arbitrary propositional CNF formula into an equivalent modal formula (the propositional formula is satisfiable iff the modal formula is satisfiable in a general model). This modal formula has some very low modal depth $h$ such that any function growing slower than a tower of 2's of height $h-5$ is a polynomial in the size of the propositional formula. The treewidth of this modal formula can be verified to be a constant. This also proves that unless Ptime $=$ NP, modal satisfiability in general models is not FPT when treewidth is the only parameter.

We can work out a composition algorithm [4, and hence conclude that with treewidth and modal depth as parameters, there is no polynomial kernel for modal satisfiability in general models.

One direction for future research is towards meta classification as done in [19], instead of the case by case analysis of this work. We can also consider variations in treewidth, such as having different domain elements representing same propositional variable at different levels in $\mathcal{S}(\phi)$. Other variations are modal circuits instead of modal formulae and generalizations of primal/dual graphs instead of incidence graphs.

Acknowledgements. The author wishes to thank Kamal Lodaya, Geevarghese Philip and Saket Saurabh for helpful discussions, pointers to related work and feedback on the draft. The author also thanks anonymous referees of a previous version of this paper for catching some subtle errors and suggesting extensions.

\section{References}

[1] A. Achilleos, M. Lampis, and V. Mitsou. Parameterized modal satisfiability. In ICALP, volume 6199 of $L N C S$, pages 369-380, 2010.

[2] I. Adler and M. Weyer. Tree-width for first order formulae. In CSL, volume 5771 of $L N C S$, pages 71-85, 2009.

[3] P. Blackburn, M. de Rijke, and Y. Venema. Modal Logic. CUP, 2001.

[4] H. L. Bodlaender, R. G. Downey, M. R. Fellows, and D. Hermelin. On problems without polynomial kernels. J. Comput. Syst. Sci., 75(8):423-434, 2009.

[5] H. L. Bodlaender and T. Kloks. Efficient and constructive algorithms for the pathwidth and treewidth of graphs. J. Alg., 21(2):358-402, 1996.

[6] H. Chen. Quantified constraint satisfaction and bounded treewidth. In R. L. de Mántaras and L. Saitta, editors, ECAI, pages 161-165. IOS Press, 2004.

[7] B. Courcelle. The monadic second-order logic of graphs III: tree-decompositions, minors and complexity issues. ITA, 26:257-286, 1992.

[8] V. Dalmau, P. G. Kolaitis, and M. Y. Vardi. Constraint satisfaction, bounded treewidth, and finite-variable logics. In $C P$ '02, pages 310-326. Springer, 2002.

\footnotetext{
${ }^{2}$ The author acknowledges an anonymous referee for pointing this out.
} 
[9] P. Enjalbert and L. F. del Cerro. Modal resolution in clausal form. Theor. Comp. Sc., 65(1):1-33, 1989.

[10] R. Fagin, J. Y. Halpern, Y. Moses, and M. Y. Vardi. Reasoning About Knowledge. MIT Press, 1995.

[11] M. Fellows, F. V. Fomin, D. Lokshtanov, F. Rosamond, S. Saurabh, S. Szeider, and C. Thomassen. On the complexity of some colorful problems parameterized by treewidth. In Comb. Opt. and Appl., volume 4616 of LNCS, pages 366-377.

[12] E. Fischer, J.A. Makowsky, and E.V. Ravve. Counting truth assignments of formulas of bounded tree-width or clique-width. Disc. App. Math., 156(4):511-529, 2008.

[13] J. Flum and M. Grohe. Parameterized Complexity Theory. Springer, 2006.

[14] E. Grädel. Why are modal logics so robustly decidable? In Current Trends in Theor. Comp. Sc., pages 393-408. 2001.

[15] M. Grohe. The structure of tractable constraint satisfaction problems. In MFCS, volume 4162 of $L N C S$, pages 58-72, 2006.

[16] J. Y. Halpern. The effect of bounding the number of primitive propositions and the depth of nesting on the complexity of modal logic. Artif. Intell., 75(2):361-372, 1995.

[17] J. Y. Halpern and Y. O. Moses. A guide to completeness and complexity for modal logics of knowledge and belief. Artif. Intell., 54(3):319-379, 1992.

[18] J. Y. Halpern and L. C. Rêgo. Characterizing the np-pspace gap in the satisfiability problem for modal logic. In IJCAI, pages 2306-2311, 2007.

[19] E. Hemaspaandra and H. Schnoor. On the complexity of elementary modal logics. In STACS, pages 349-360, 2008.

[20] A. Herzig and J. Mengin. Uniform interpolation by resolution in modal logic. In JELIA '08, volume 5293 of LNCS, pages 219-231, 2008.

[21] U. Hustadt and R. A. Schmidt. An empirical analysis of modal theorem provers. J. Applied Non-Classical Logics, 9(4), 1999.

[22] Y. Kazakov and I. Pratt-Hartmann. A note on the complexity of the satisfiability problem for graded modal logics. In $L I C S$, pages 407-416, 2009.

[23] R. E. Ladner. The computational complexity of provability in systems of modal propositional logic. SIAM J. Comput., 6(3):467-480, 1977.

[24] D. Marx. Can you beat treewidth? In FOCS, pages 169-179, 2007.

[25] L. A. Nguyen. On the complexity of fragments of modal logics, volume 5 of Advances in Modal logic, pages 249-268. 2005.

[26] G. Pan and M. Y. Vardi. Optimizing a BDD-based modal solver. In CADE-19, volume 2741 of $L N C S$, pages 75-89. 2003.

[27] G. Pan and M. Y. Vardi. Fixed-parameter hierarchies inside pspace. In LICS '06, pages 27-36. IEEE Computer Society, 2006.

[28] V. R. Pratt. Application of modal logic to programming. Studia Logica, 39(2-3):257-274, 1980.

[29] J. H. Reif and A. P. Sistla. A multiprocess network logic with temporal and spatial modalities. In $I C A L P$, volume 154 of $L N C S$, pages 629-639, 1983.

[30] M. Samer and S. Szeider. Constraint satisfaction with bounded treewidth revisited. J. Comput. Syst. Sci., 76(2):103-114, 2010.

[31] B. ten Cate. A note on the expressibility problem for modal logics and star-free regular expressions. Inf. Process. Lett., 109(10):509-513, 2009.

[32] M. Thorup. All structured programs have small tree width and good register allocation. Inf. and Comput., 142(2):159 - 181, 1998.

[33] M. Y. Vardi. Why is modal logic so robustly decidable? In Descriptive Complexity and Finite Models, pages 149-184. AMS, 1996. 